\title{
Three-Dimensional Culture and FGF Signaling Drive Differentiation of Murine Pluripotent Cells to Distal Lung Epithelial Cells
}

\author{
Emily Fox, ${ }^{1,2}$ Sharareh Shojaie,, Jinxia Wang, ${ }^{1}$ Irene Tseu, ${ }^{1,}$ Cameron Ackerley, \\ Mélanie Bilodeau, ${ }^{3}$ and Martin Post ${ }^{1,2}$
}

Reciprocal signaling between the lung mesenchyme and epithelium is crucial for differentiation and branching morphogenesis. We hypothesized that the combination of signaling pathways comprising early epithelialmesenchymal interactions and a 3D spatial environment are necessary for an efficient induction of embryonic and induced pluripotent stem cells (ESCs and iPSCs) into a lung cell phenotype with hallmarks of the distal niche. Aggregating early, but not late, embryonic lung mesenchyme with endoderm-induced mouse ESCs and iPSCs for 6 days resulted in organization into tubular structures and differentiation of the tubular lining cells to an NKX $2-1^{+} / \mathrm{SOX}^{-} / \mathrm{SOX}^{+} / \mathrm{proSFTPC}^{+}$lineage. Over $80 \%$ of the endoderm-induced cells committed to an NKX2 $-1^{+}$lineage. Electron microscopy analysis demonstrated numerous multivesicular bodies and glycogen deposits in the tubular lining cells, characteristic features of type II epithelial cell progenitors. Using soluble FGFR2 receptor antagonists, we demonstrate that reciprocal fibroblast growth factor (FGF) 2, 7, and 10 signaling is essential for differentiation of endoderm-induced cells to an NKX2-1 ${ }^{+} /$proSFTPC $^{+}$phenotype within 3D aggregates. Only FGF2 was able to commit endoderm-induced cells in monolayer cultures to an NKX2 $-1^{+}$lineage, however with a significant lower efficiency $(\sim 16 \%)$ than seen with mesenchyme. Thus, while FGF2 signaling alone can induce a primed population of ESCs and iPSCs, the cells do not differentiate to distal lung epithelial progenitors with the same efficiency and level of maturity that is achieved when the complex tissue and 3D environment of the developing lung is more accurately recapitulated.

\section{Introduction}

$\mathrm{T}$ HE MAMMALIAN LUNG DEVELOPS as an out-pouching of the foregut at embryonic day 9.5 (E9.5) in the mouse and responds to cues in the local environment, primarily from the adjacent mesenchyme (Mes) [1]. As the lung continues to develop, crosstalk between the epithelial and mesenchymal layers establishes the morphogenesis and proximal-distal patterning that result in anatomically and physiologically distinct areas of the lung [2-4]. Given the complexities of the crosstalk occurring during lung development, it is no surprise that the complete compliment of factors and the precise timing and dosage required for lung specification has yet to be elucidated. While other endodermal organs, such as the liver and pancreas, have seen success in producing relatively pure populations of progenitor cells capable of expansion in culture [5-7], this has yet to be achieved in the lung. Various studies with embryonic stem cells (ESCs) have shown that these cells can be encouraged to differentiate into different lung cell types, including alveolar type II cells [8-15] and bronchiolar ciliated cells and Clara cells [16-18]. Similar findings have been obtained with induced pluripotent stem cells (iPSCs) $[17,19,20]$. Many of these studies achieved distal lung differentiation with very low efficiencies $(<3 \%)$, which could be considered a result of spontaneous differentiation, while more successful protocols with iPSCs use undefined culture conditions [19], providing no further insight as to how the differentiation is occurring. The most effective ESC [11,12,17] and iPSC [21,22] studies with respect to efficiency using defined factors have attempted to recapitulate what occurs during development in vivo in a stepwise fashion, starting with endoderm specification via the nodal pathway [23-25] followed by anterior endoderm induction and subsequent lengthy stimulation with a mixture of growth factors implicated in lung development. Differentiation efficiencies and duration of differentiation in vitro could be improved, however, if the in situ microenvironment of epithelialmesenchyme interactions, including the $3 \mathrm{D}$ matrix milieu, is better recapitulated.

\footnotetext{
${ }^{1}$ Physiology and Experimental Medicine Program, Peter Gilgan Centre for Research and Learning, Hospital for Sick Children, Toronto, Ontario, Canada.

${ }^{2}$ Department of Physiology, University of Toronto, Toronto, Ontario, Canada.

${ }^{3}$ Developmental and Stem Cell Biology Program, Peter Gilgan Centre for Research and Learning, Hospital for Sick Children, Toronto, Ontario, Canada.
} 
To gain further insight into the mechanisms of commitment from endoderm to early lung epithelial progenitor cells, we used ESCs and iPSCs to model lung development. We took advantage of the epithelial-mesenchymal interactions that are crucial for lung morphogenesis and differentiation by using early embryonic lung mesenchyme, producer of various growth and matrix factors implicated in early lung development, to differentiate ESCs and iPSCs to a lung epithelial cell phenotype. We demonstrate that early (E13), but not late (E19), distal embryonic lung mesenchyme of mouse and rat possesses all the instructive cues necessary to drive the majority of endoderm-induced mouse ESCs and iPSCs to an early proSFTPC $^{+}$lung epithelial phenotype in a 3D culture environment. Growth factor receptor inhibition studies revealed that signaling via FGFR1c and/or FGFR2c induces early lung epithelial $\left(\mathrm{NKX}_{2}-1^{+}\right)$differentiation in the 3D aggregates while further advancement to a NKX2-1 ${ }^{+} /$pro$\mathrm{SFPTC}^{+}$phenotype requires signaling via FGFR2b. Initial commitment of endoderm-induced mouse ESCs to an NKX2$1^{+}$lineage was achieved with fibroblast growth factor-2 (FGF2), but not FGF9 treatment; however, the differentiated cell population was immature and less numerous compared with that generated using lung mesenchyme in 3D culture.

\section{Materials and Methods \\ ESC/iPSC maintenance}

The double-reporter mouse ESC line Foxa2/hCD4;Bry/ GFP was obtained from Dr. Gordon Keller and was maintained as described previously [6]. The mouse iPSC line iPS EOS3F-29 was obtained from Dr. James Ellis and was maintained as described [26]. Additional ES and iPS cell lines tested produced similar results (Supplementary Fig. S2D; Supplementary Data are available online at www.liebertpub .com/scd). These include the R1 line [27], C2 line [28], iPS EOS3F28 [29], and WT3\#6 line (J. Ellis, unpublished data). An Nkx2-1 ${ }^{\text {mCherry }}$ reporter ESC line was used for a subset of experiments to allow for tracking and quantification of Nkx2-1 expression [30].

\section{ESC/iPSC endoderm differentiation}

ESCs were trypsinized using TrypLE express (Gibco, Burlington, ON, Canada) for $4 \mathrm{~min}$ at $37^{\circ} \mathrm{C}$ and cultured at 20,000 cells $/ \mathrm{mL}$ in low-adherence six-well plates (Nunc, Roskilde, Denmark) to allow for embryoid body (EB) formation. EBs were collected and dissociated after $48 \mathrm{~h}$ and reaggregated with $50 \mathrm{ng} / \mathrm{mL}$ activin A (R\&D Systems, Minneapolis, MN) for an additional $48 \mathrm{~h}$. EBs were harvested for flow cytometry or fluorescence-activated cell sorting (FACS) analysis. iPSCs were differentiated in a similar manner with one additional day of activin A treatment. To achieve anterior definitive endoderm differentiation, sorted endoderm cells were seeded on a monolayer and treated with $200 \mathrm{ng} / \mathrm{mL}$ NOGGIN (BMP signaling inhibitor) and $10 \mu \mathrm{M} \mathrm{SB}-431542$ (pharmacological inhibitor of TGF $\beta$ / nodal signaling) for $24 \mathrm{~h} \mathrm{[31].}$

\section{FACS and flow cytometry}

Day-4 (ESCs) or day-5 (iPSCs) EBs were dissociated and the single-cell suspensions were labeled with antibodies in
Hank's balanced salt solution (HBSS) supplemented with $2 \%(\mathrm{v} / \mathrm{v})$ fetal bovine serum (FBS) and $10 \mathrm{mM}$ HEPES buffer (Invitrogen, Burlington, ON, Canada) for $30 \mathrm{~min}$ on ice. Antibodies used are summarized in Supplementary Table S1. Cells were analyzed using the Galios flow cytometer and data analysis was carried out using Kaluza software (Beckman Coulter, Mississauga, ON, Canada). Cell sorting was carried out on AriaII-RITT BRV (Becton Dickson, Franklin Lakes, NJ) and MoFlo BRU cell sorter (Beckman Coulter).

\section{ESC/iPSC-lung mesenchyme aggregate cultures}

All animal experiments were approved and carried out in accordance with the animal care committee guidelines of the Hospital for Sick Children. CD1 mice were time-bred onsite overnight while timed-pregnant Wistar rats were ordered from Charles River (St. Constant, QC, Canada). On day 11.5 (mouse) or 13 (rat) of gestation (day 19 for late mesenchyme), dams were euthanized and fetuses were delivered by caesarean section. Lungs were dissected from embryos and transferred to HBSS and treated with $20 \%$ (v/v) dispase and $1.5 \%$ (v/v) DNase (Invitrogen, Mississauga, $\mathrm{ON}$, Canada) for $45 \mathrm{~min}$ at $37^{\circ} \mathrm{C}$. Cultures were neutralized with Dulbecco's modified Eagle's medium $+10 \%$ (v/v) FBS (Invitrogen) and the distal mesenchymal layer was separated manually from the endoderm using 27-gauge needles. Distal mesenchyme was transferred to Nucleopore membranes (8- $\mu \mathrm{m}$ pore size; Whatman, Piscataway, NJ) with 10-20 activin-induced $\mathrm{EBs}$, resulting in a ratio of $1: 4 \mathrm{EBs}$ to mesenchyme. These aggregates and controls (EBs without mesenchyme) were cultured in serum-free differentiation medium, at air-liquid interface (Supplementary Fig. S1). Cells were maintained in culture for a total of 6 days with two media changes. To confirm absence of endodermal carryover during mesenchyme dissection, aggregates using ES-EBs and mesenchyme from E11.5 enhanced green fluorescent protein (EGFP) mice were generated and cultured. This resulted in no EGFP expression in epithelial tubular structures, consistent with no endodermal carryover during the dissection (Supplementary Fig. S2A). Alternatively, ES-EB-expressing RFP variant, DsRed.T3 (gift of Dr. A. Nagy, Lunenfeld Research Institute, Toronto, Canada), was aggregated and cultured with E13 rat mesenchyme. Coexpression of this RFP variant with early lung endodermal marker NKX-2.1 confirmed absence of endodermal carryover (Supplementary Fig. S2B). For the inhibition studies, soluble receptor-Fc chimeras were added to mesenchyme and EBs on day 0 of culture for $1 \mathrm{~h}$ prior to aggregation in the following concentrations: $100 \mathrm{ng} / \mathrm{mL}$ sFGFR2c, $650 \mathrm{ng} / \mathrm{mL}$ sFGFR2b, and $500 \mathrm{ng} / \mathrm{mL}$ sNGFR (R\&D Systems, Burlington, ON, Canada). These were the lowest dosages to elicit a response. On day 6, the aggregates and controls were removed from culture and analyzed. Soluble receptor neutralization experiments using FGFs were carried out in the same manner as described previously, with the preincubation of soluble receptors with either $500 \mathrm{ng} / \mathrm{mL}$ FGF2, FGF10, or FGF7 to ensure complete saturation.

\section{Immunofluorescence}

Immunofluorescence analysis was performed as described previously [32]. Details of antibodies are provided in 
Supplementary Table S1. Whole lungs of E16 mouse embryos were used as positive controls. Images were captured with a Leica CTRMIC 6000 confocal microscope in conjunction with a Hamamatsu C910013 spinning-disc camera (Leica Microsystems, Inc., Concord, ON, Canada).

\section{Electron microscopy}

For transmission electron microscopy (TEM), specimens were fixed in $2.5 \%(\mathrm{w} / \mathrm{v})$ glutaraldehyde in $0.1 \mathrm{M}$ phosphate buffer ( $\mathrm{pH}$ 7.4) for $4 \mathrm{~h}$ and processed as previously described [13]. For immunogold EM, specimens were fixed in $4 \%(\mathrm{v} / \mathrm{v})$ paraformaldehyde and $0.1 \%(\mathrm{v} / \mathrm{v})$ glutaraldehyde in $0.1 \mathrm{M}$ phosphate buffer ( $\mathrm{pH}$ 7.4) for $4 \mathrm{~h}$ and processed as previously described [33]. Cryosections were incubated with primary antibodies for $1 \mathrm{~h}$ (Supplementary Table S1) followed by incubation with 10-nm gold-goat anti-rabbit IgG complexes (Amersham, Life Sciences, Arlington Heights, IL) for an additional hour at room temperature. Controls included omission of the primary antisera. All EM samples were examined on a JEOL JEM 1011 transmission electron microscope (JEOL USA, Peabody, MA).

\section{Quantitative polymerase chain reaction}

RNA was isolated from ESC/iPSC-mesenchyme aggregates using the Arcturus Picopure RNA isolation kit (Life Technologies, Burlington, ON, Canada) using an RNasefree DNase I treatment step (Qiagen, Toronto, ON, Canada). One microgram of total RNA was reverse transcribed with random hexamers using the Superscript III enzyme according to the manufacturer's specifications (Invitrogen). Ten micrograms of template cDNA (1 ng for $18 \mathrm{~S}$ ) was used for real-time polymerase chain reaction (PCR) using SYBR GreenER quantitative PCR (qPCR) SuperMix in conjunction with murine-specific primers sets (Supplementary Table S2). qPCR was performed using OneStepPlus qPCR (Applied Biosystems, Burlington, ON, Canada). Fold change was calculated with normalization to $18 \mathrm{~S}$, according to Livak and Schmittgen [34].

\section{Immunoblotting}

Immunoblotting was performed as described previously [32]. Antibodies used are listed in Supplementary Table S1.

\section{Quantification of structures}

Luminal area of the tubular structures was measured using Image J version 1.44p (National Institute of Health, Bethesda, MD). Five randomly selected nonoverlapping fields from sections obtained from four aggregates per group were counted and total luminal epithelial area per aggregate consisted of these pooled counts normalized against total field area. Each field was viewed at 200 times magnification.

\section{Statistical analysis}

Data are expressed as means \pm SEM. Student's unpaired $t$-test and one-way analysis of variance with Holm-Sidak post hoc analysis were performed using SigmaPlot version
11 software (Systat Software, San Jose, CA). $P<0.05$ was deemed statistically significant.

\section{Results \\ Embryonic lung mesenchyme directs ESCs and iPSCs to an early distal lung phenotype}

Crosstalk between the mesenchyme and the endoderm is critical for establishing cell fate and maturation in the developing lung [3]. While lung mesenchyme has been previously used to induce ESCs to a lung cell phenotype [9], its induction potential has not been tested on a pure endoderm population derived from ESCs, nor has it been attempted with iPSCs. To determine the mesenchymal signals necessary for commitment to lung, we first combined endoderminduced EBs derived from ESCs (ES Foxa2/hCD4;Bry/ GFP) or iPSCs (iPS EOS3F-29) with early distal lung mesenchyme to assess its induction capacity (Fig. 1A and Supplementary Fig. S1). Following activin A treatment, $\sim 50 \%$ of ES-EB and iPS-EB cultures coexpressed definitive endoderm markers CXCR4 and cKIT. Roughly half of the ES-EB cells $(\sim 51 \%)$ also coexpressed GFP and hCD4, surrogate markers for BRY and FOXA2 in this ESC line, respectively (data not shown). To obtain a pure population of endodermal cells devoid of remaining pluripotent cells and nonendoderm lineages, ES-EBs and iPS-EBs were sorted for CXCR4 and EPCAM following activin treatment, prior to aggregation with mesenchyme ( $\mathrm{P} 4$ population, Fig. 1B). CXCR4 is expressed in the embryonic endoderm and mesoderm [35], while EPCAM is expressed in embryonic endoderm and extraembryonic endoderm [36]. Sorted cells form endoderm cell clusters when cultured under nonadherent conditions and express foregut endodermal markers FOXA2 and SOX2 following 6 days of culture at an air-liquid interface, but do not express lung markers NKX21 and proSFTPC (Fig. 1C). When sorted ES-EBs and iPSEBs were combined with early lung mesenchyme, both cell lines organized into tubular structures with the lining cells coexpressing NKX2-1 and SOX9, characteristic of the distal tip progenitors that generate alveolar epithelial lineages, reminiscent of SOX9 expression in natural E14.5 mouse lung tissue [37]. Lack of SOX2 and Club/Clara cell marker SCGB1A1 expression indicates absence of proximal airway lineage within the aggregate cultures [38] (Fig. 1C). Coexpression of proSFTPC and SOX9 provides further evidence that a distal lung progenitor population has been established [21] (Fig. 1D).

Colocalization of Foxa2-hCD4 and NKX2-1 in the ESCmesenchyme aggregates demonstrated that the tubular epithelial lining cells were derived from the ESC population (Supplementary Fig. S2C). In addition to the distal lung markers, the aggregate cultures were stained for keratin 5 (basal cell marker), $\beta$ tubulin IV (ciliated cell marker), and Pgp9.5 (neuroepithelial cell marker), all of which are proximal lung epithelial markers [39-41]. None were found to be present in the ESC- or iPSC-mesenchyme aggregate cultures. Positive controls included lung samples from various developmental gestations (Supplementary Fig. S3).

TEM analysis was performed to characterize the organization and differentiation of the ESCs and iPSCs within the aggregates at the ultrastructural level. Endoderm-induced 
cells cultured with mesenchyme oriented into tubular structures with a clear lumen, surrounded by mesenchymal cells (Fig. 2A). Cells lining the tubules appeared columnar, polarized, and with microvilli on the apical surface. Extracellular matrix was found on the basal surface of the structures, typical of basement membrane (Supplementary Fig. S4E). Many of the cells in the tubules contained large glycogen deposits (Supplementary Fig. S4D), structures reminiscent of multivesicular bodies (Fig. 2B, left panel and Supplementary Fig. S4A, B) and prelamellar bodies (Fig. 2B, right panel), characteristic features of immature distal type II epithelial cells. Immunogold labeling of these aggregates (Fig. 2C) verified that proSFTPC localized within the multivesicular structures of the epithelial lining cells, in line with reported localization of proSFTPC in maturing alveolar type II epithelial cells [42]. Immunogold labeling of TJP1 (also known as ZO-1) demonstrated the presence of tight junctions between the epithelial cells, hallmark of a functional epithelial lining (Fig. 2C). In contrast, EBs cultured without mesenchyme at an air-liquid interface contained poorly organized cells with little or no tubule formation. Many of the cells had apoptotic nuclei and displayed poor contact with one another (Fig. 2A, inset).

Markers of other endodermal organ lineages as well as Oct4, a pluripotency marker, and Olig2, a neuronal marker that is coexpressed with $N k x 2-1^{+}$cells in the developing forebrain, were examined in the aggregates to determine whether the combination with early lung mesenchyme prevented the formation of other lineages. Real-time PCR analysis showed absence of expression of other endoderm lineage markers ( $P a x 8$ for thyroid, $P d x 1$ for pancreas, and Albumin for liver) in EB-mesenchyme aggregates using sorted endoderm-induced ESCs or iPSCs when compared with respective tissues or stem cell controls (Supplementary Fig. S5A). Further, sorting of iPSCs for an enriched endoderm population prior to combination with mesenchyme increased the expression of both $N k x 2-1$ and surfactant protein B $(S f t p b)$, compared with nonsorted aggregate cultures. Aggregates exhibited significantly greater expression of Nkx2-1, Sftpb, and Sftpc than E13 lung controls, suggesting that they comprise a higher proportion of distal lung epithelial cells or they represent a later stage of differentiation (Supplementary Fig. S5B, C).

As a surrogate assessment of epithelial branching, we measured the luminal area of the airways and tubular structures per unit field in the developing lung (E13, E16, and E18) and EB + Mes and iPS + Mes aggregates, respec- tively. The measured luminal epithelial area of both ESCand iPSC-derived mesenchyme aggregates at day 6 resembled that of E18 mouse lungs (Supplementary Fig. S5D), suggesting extensive epithelial surface expansion by tubular branching in the aggregates. Immunostaining at several time points reveals the presence of NKX2-1- and proSFTPCpositive tubular structures in day-4 aggregates resembling that of the developing lung (Supplementary Fig. S5E). The increase in number of positive tubular structures at day 6 compared with day 4 suggests ongoing branching morphogenesis with extended time culture.

While rat mesenchyme was used in the experiments represented here, these results were reproducible using mouse lung mesenchyme (Supplementary Fig. S6A, B). This underlies the conserved nature of the signaling pathways involved in early lung commitment. In addition to culturing EB alone as control, aggregates were generated using E13 rat skin mesenchyme. The absence of lung lineage differentiation in these aggregates provided evidence for the specificity in the process of lung epithelial induction by lung mesenchyme (Supplementary Fig. S6C).

\section{Highly efficient induction of an NKX2-1 ${ }^{+}$population is achieved within the epithelial cell compartment}

To test the efficiency of NKX2-1 induction by early lung mesenchyme, we used an $\mathrm{Nkx} 2-1^{\text {mCherry }} \mathrm{ES}$ cell line to quantify NKX2-1 ${ }^{+}$progenitors generated in the mesenchyme aggregates [30]. These ESCs were induced as described before and sorted for dual expression of CXCR4 and EPCAM (Fig. $3 \mathrm{~A})$. As expected aggregation with early lung mesenchyme resulted in organization into tubular structures with lining cells coexpressing NKX2-1 and proSFTPC (Fig. 3B). To quantify the proportion of cells committed to a lung epithelial cell lineage, we first examined the population that expressed EPCAM. Following 6 days in culture, $20.85 \% \pm 1.8 \%$ (mean \pm $\mathrm{SE}, n=7$ ) of the total cells in the $3 \mathrm{D}$ aggregates were EP$\mathrm{CAM}^{+}$by flow cytometry, retaining the initial fraction of around $25 \%$ endodermal cells used to generate the aggregates (Fig. 3C). Flow cytometry was performed for mCherry expression and was found to be $16.5 \% \pm 1.6 \%$ (mean \pm SE, $n=7)$ of the total cells, suggesting that the aggregation of endodermsorted ESCs with early lung mesenchyme had resulted in $\sim 80 \%$ of the $\mathrm{EPCAM}^{+}$epithelial cells to commit to an NKX2 $-1^{+}$lineage (Fig. 3C). To corroborate these findings, we repeated the experiment with Foxa2/hCD4;GFP/Bry ES cells to track the portion of $\mathrm{EPCAM}^{+}$cells that were also

FIG. 1. Embryonic lung mesenchyme stimulates differentiation of ESC- and iPSC-derived endoderm to an NKX2-1/ proSFTPC-positive lineage. (A) A schematic of the experimental design. Aggregates are generated using sorted ESC- or iPSCderived endoderm with E13 embryonic lung mesenchyme. (B) Fluorescence-activated cell sorting for CXCR4 ${ }^{+} / \mathrm{EPCAM}^{+}$of ESCs and iPSCs following activin A treatment. The sorted population of cells is marked by the "P4" gate. (C) Immunostaining for markers of lung epithelial progenitors. Top panel represents the EBs cultured alone and is representative for both cell lines. FOXA2 (green) and NKX2-1 (green) are expressed in the cells lining the tubules in the 3D aggregates. SOX2 (red) is not expressed in NKX2-1 ${ }^{+}$structures, while SOX9 (red) is coexpressed at budding regions of the NKX2-1 ${ }^{+}$structures in both ES and iPS cell lines cultured with mesenchyme, in agreement with the natural E14.5 mouse lung. NKX2-1 (green) is also coexpressed with proSFTPC (red) while SCGB1A1 (red) was absent in both ES and iPS cell lines. Scale in upper left panel is representative of all images. (D) Immunofluorescent staining for distal lung epithelial progenitors. Distal tubules immunopositive for SOX9 (red) coexpress proSFTPC (green) in both ES and iPS cell lines cultured with mesenchyme, similar to natural E14.5 mouse lung. Scale in right panel is representative of all images. EBs, embryoid bodies; ESC, embryonic stem cell; iPSC, induced pluripotent stem cell; Mes, mesenchyme. Color images available online at www.liebertpub.com/scd 
A

ES-EB day4 iPS-EB day 5

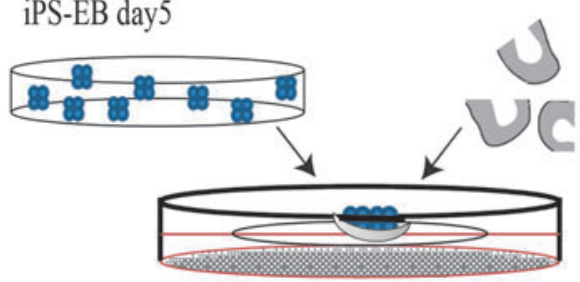

air-liquid interface
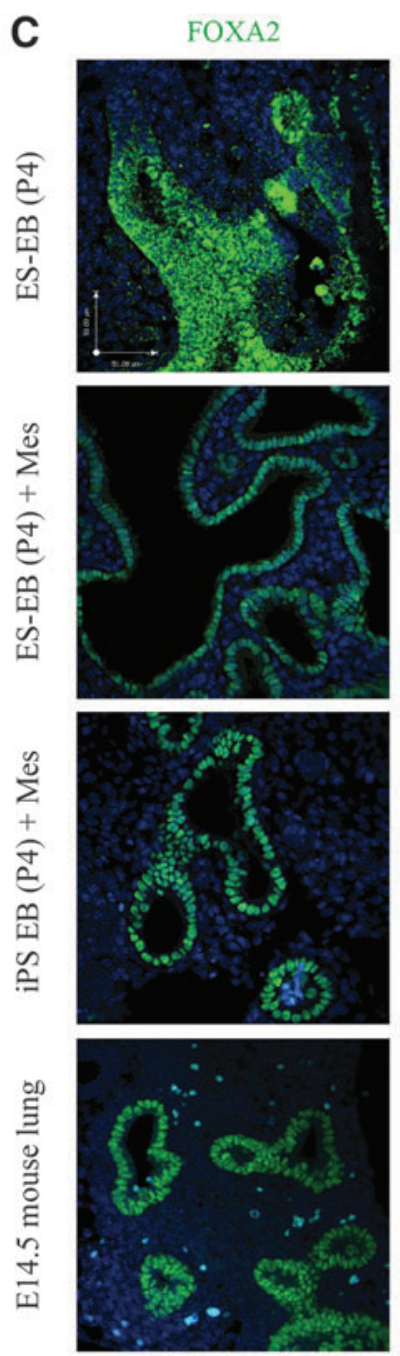

D $\quad$ ES-EB (P4) + Mes

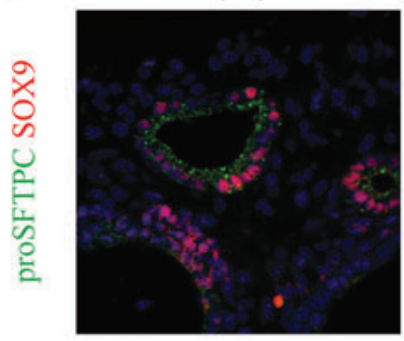

NKX2-1 SOX2
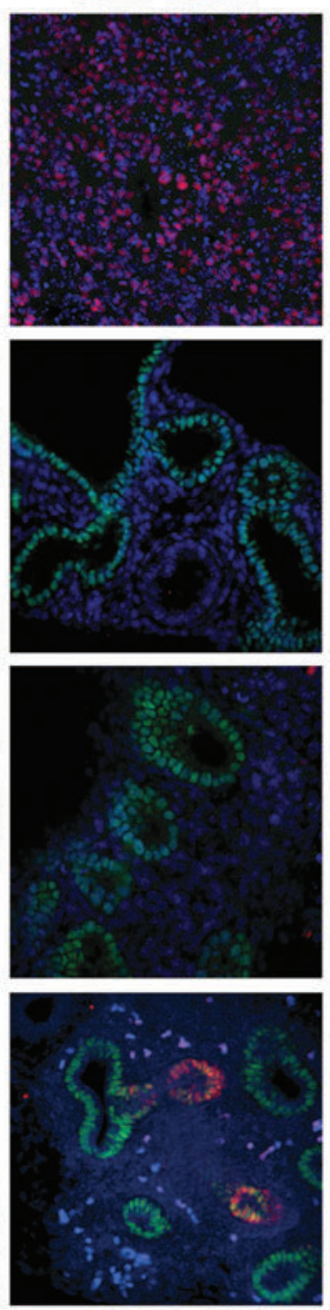

iPS-EB (P4) + Mes

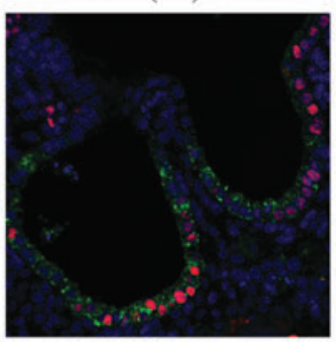

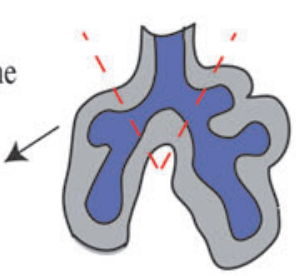

E13 rat lung

NKX2-1 SOX9
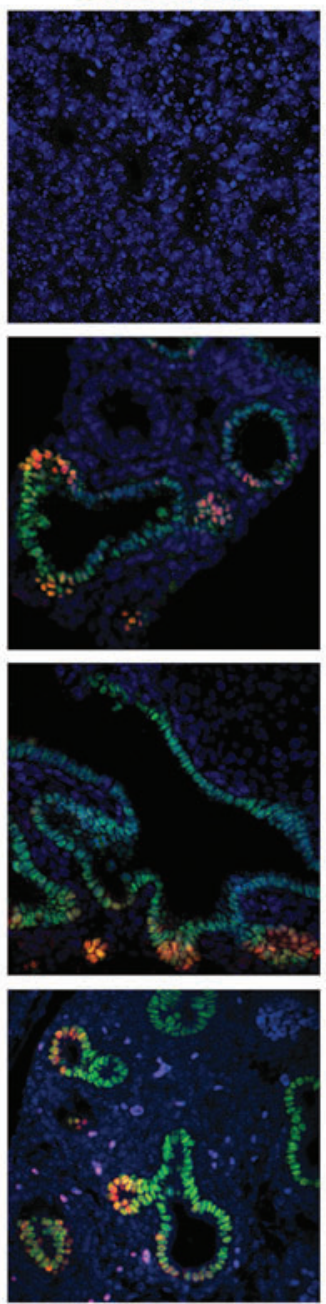

E14.5 mouse lung

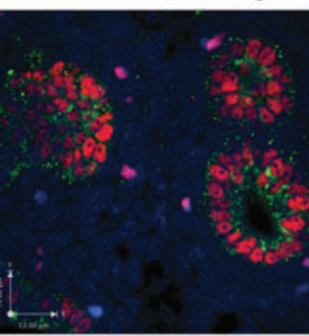

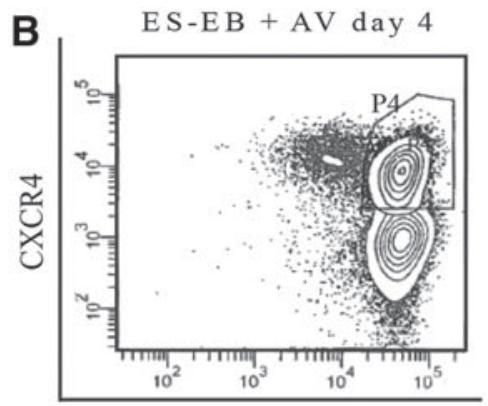

Epcam

NKX2-1 proSFTPC

NKX2-1 SCGB1A1
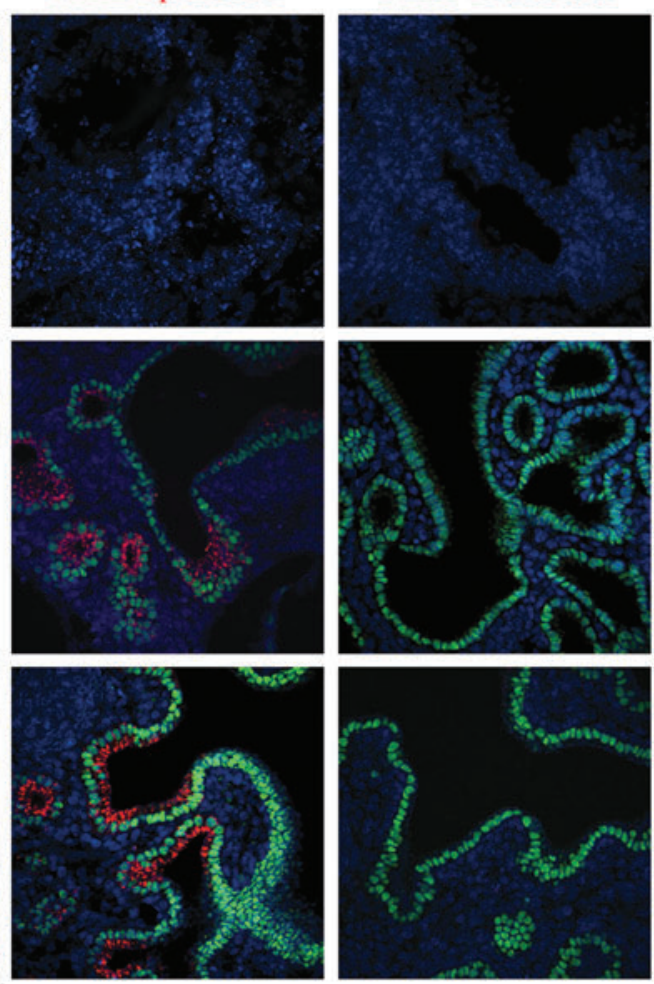
A

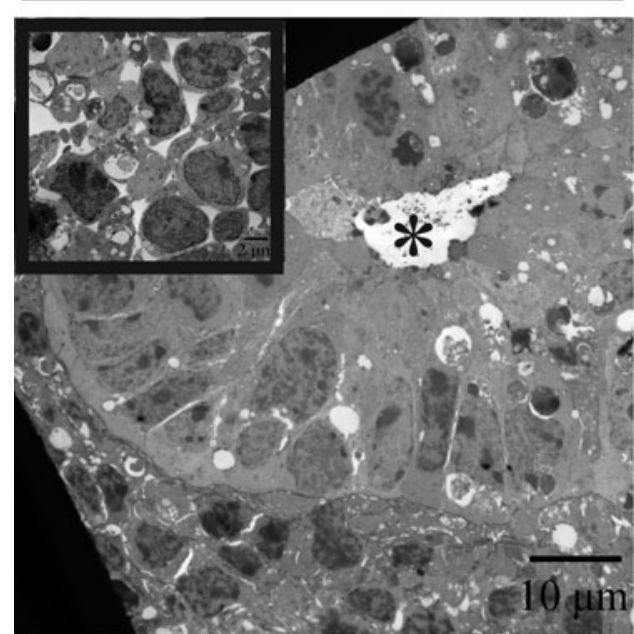

FIG. 2. Ultrastructural detail of ESC- and iPSC-derived NKX2-1/proSFTPC-positive lineage. (A) TEM of mesenchyme-EB aggregates. A representative of $\mathrm{EBs}$ cultured without mesenchyme is shown in inset. EBs aggregated with mesenchyme show organization into a polarized epithelial layer. Lumen (asterisk). (B) tivesicular bodies and immature lamellar bodies found in epithelial cells lining the tubules. (C) Immuno-EM for proSFTPC and TJP1. Multiare immunogold positive for proSFTPC (black arrowheads). Tight junctions show positive immunogold staining for TJP1 (black arrowheads). $\mathrm{AV}$, activin A; TEM, transmission electron microscopy.

\section{B} Representative image of mulvesicular/prelamellar bodies

\begin{abstract}
$\mathrm{ES}+\mathrm{AV}+\mathrm{Mes}$
\end{abstract}
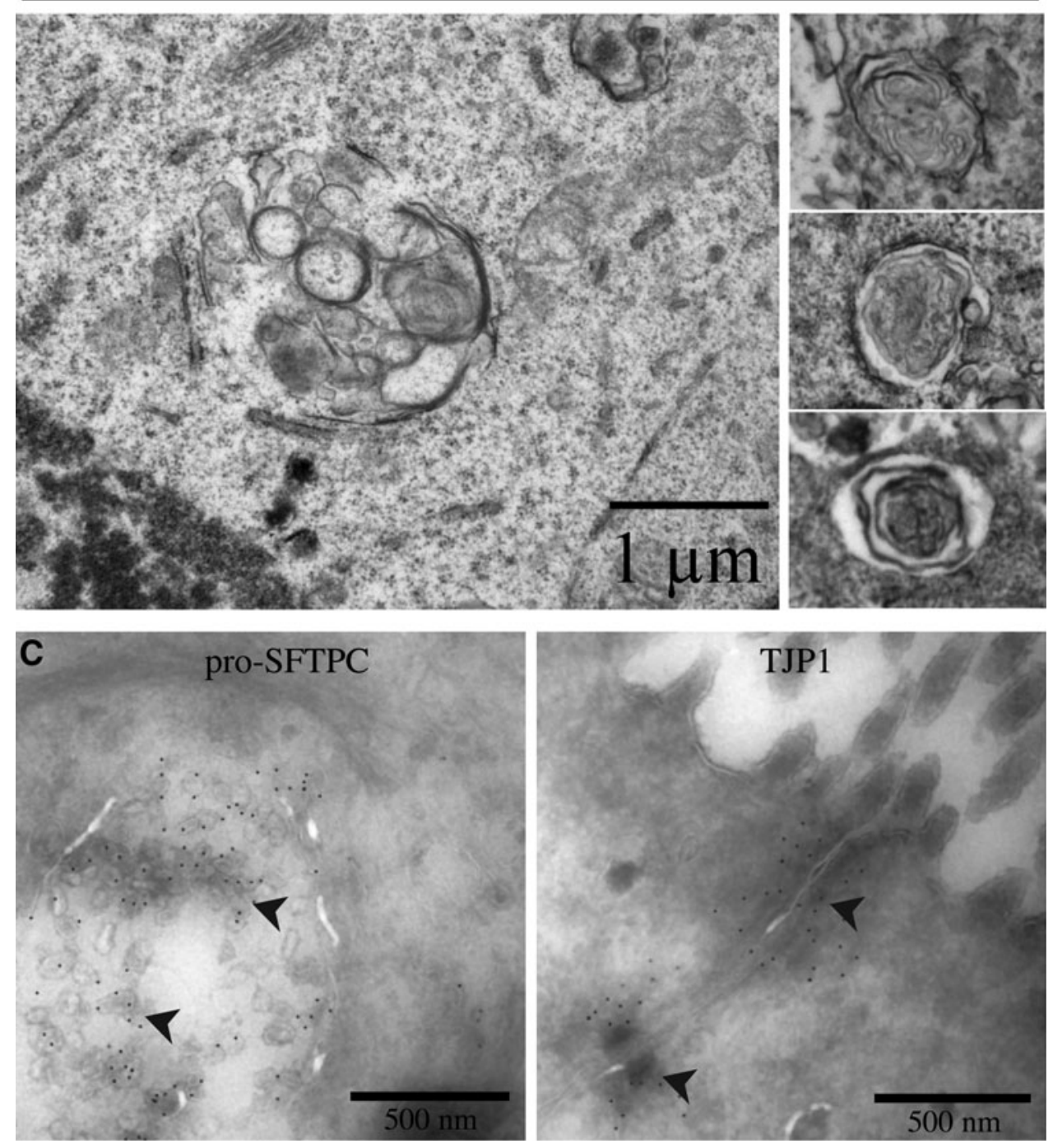

expressing hCD4, a surrogate marker for FOXA2 in this cell line. Following 6 days of culture at air-liquid interface, we found $28 \%$ of the total cells in the EB-mesenchyme aggregates to coexpress EPCAM and hCD4. Since 35\% of the total cell population was $\mathrm{EPCAM}^{+}$, these results demon- strate that about $80 \%$ of the epithelial cells were positive for FOXA2 (assessed by hCD4 expression) (Fig. 3D). Immunostaining of these aggregate cultures also demonstrated colocalization of EPCAM and NKX2-1 in the epithelial cells lining the tubule structures (Fig. 3E). Overall, these 

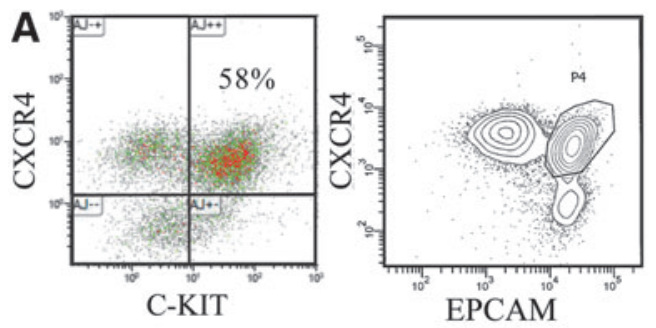

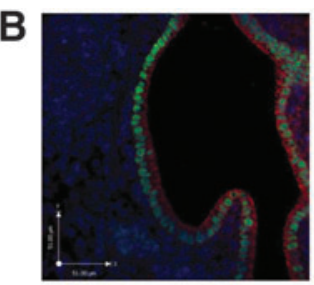

NKX2-1EPCAM

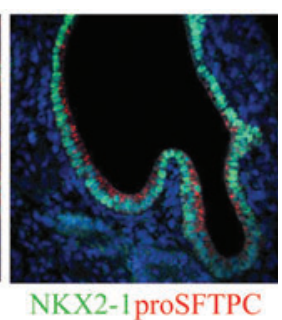

NKX2-1 proSFTPC
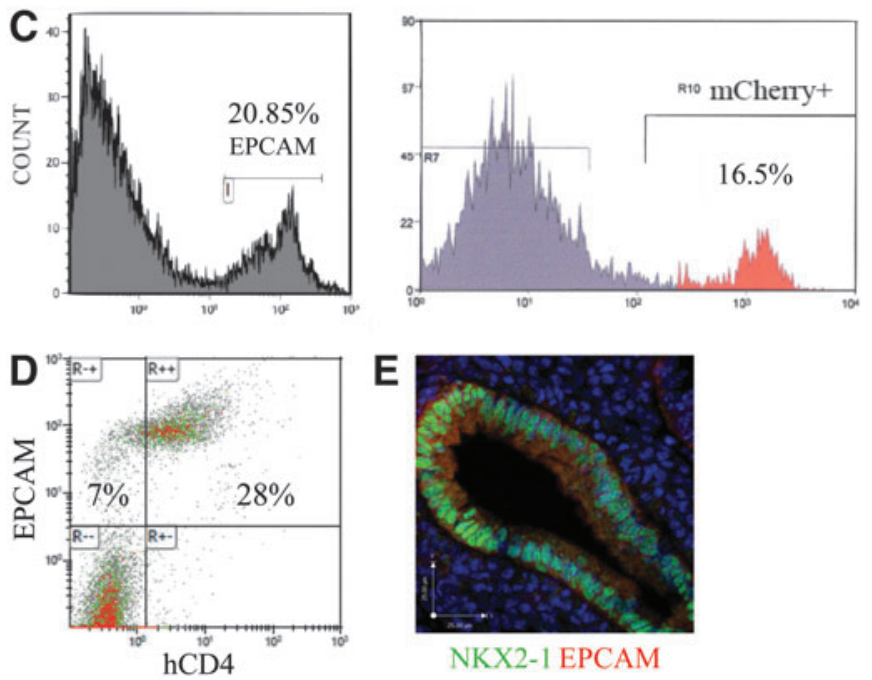

FIG. 3. Quantification of NKX2-1 ${ }^{\text {mCherry }}$-positive ESCs in aggregate cultures. (A) Flow cytometry analysis showed that differentiation of the NKX2-1 ${ }^{\text {mCherry }}$ ESCs into endoderm occurs with similar efficiency ( 58\%) as the Foxa2/hCD4;Bry/ GFP cell line. P4 represents the EPCAM/CXCR4-positive endoderm population sorted for aggregation with mesenchyme. (B) Immunostaining for NKX2-1 and proSFTPC of the sorted NKX2-1 ${ }^{\text {mCherry }}$ ES-EB containing aggregate cultures revealed a similar pattern of expression (right) as shown previously for the Foxa2/hCD4;Bry/GFP ES cell line (Fig. 1). NKX2-1 and EPCAM colocalize within the tubular structures. (C) Flow cytometry for mCherry following trypsinization of the EBmesenchyme aggregates demonstrates that $\sim 16 \%$ of the total cells express NKX2-1 (right) while EPCAM (left) is expressed in $\sim 21 \%$ of the total cells. (D) Flow cytometry of similar aggregates using Foxa2/hCD4;Bry/GFP ES-EB shows that $\sim 35 \%$ of the total cells in the EB-mesenchyme aggregates express EPCAM (upper half) while $28 \%$ of the total cells coexpress EPCAM and hCD4 (upper right quadrant). (E) Immunostaining demonstrates colocalization of NKX2-1 and EPCAM population within tubular structures in these aggregates (representative images). Color images available online at www.liebertpub.com/scd

results demonstrated that the three-dimensional aggregation system leads to the rapid generation of organized pulmonary epithelial cells with high efficiency.

\section{Late lung mesenchyme is unable to commit ESCs or iPSCs to a lung cell phenotype}

Previously we have shown that late (E19) rat lung mesenchyme is a stronger morphogenic inducer of E13 rat lung epithelium compared with early (E13) mesenchyme [32]. To determine whether late mesenchyme has similar inductive effects on endoderm-induced ESCs and iPSCs, aggregate cultures were generated using late mesenchyme [32]. Immunostaining of these cultures showed no evidence of NKX2-1 or proSFTPC at the protein level (Fig. 4A). Recombinants, composed of E13 lung epithelium and E19 mesenchyme, were generated as previously described [32] and functioned as controls. As expected, results from these control experiments showed that combining late mesenchyme with early epithelium produces structures with saccular, lung-like morphology coexpressing NKX2-1 and proSFTPC (Fig. 4B) [32]. To elucidate the difference be- tween early and late mesenchyme that is accountable for this contrast in induction potential of endoderm-induced ESCs and iPSCs, we examined the expression of selected FGFs implicated in lung development $[1,43]$ between the E13 and E19 mesenchyme. Real-time PCR analysis revealed that expression of $F g f 2$ and $F g f 10$ mRNA was significantly greater in early versus late mesenchyme (Fig. 4C). Conversely, Fgf7 mRNA levels dramatically increased with advancing gestation in accordance with previously published results [44]. This suggests that FGF2 and/or FGF10 in early mesenchyme are candidate mediators of commitment to an NKX2-1 ${ }^{+}$phenotype.

The interaction between ESCs and iPSCs with early lung mesenchyme was further characterized by evaluating whether the mesenchyme continued to develop alongside the endoderm-induced cells for the duration of aggregate culture. We observed that the level of $F g f 2$ mRNA in both ES and iPS aggregates peaked at day 4 and declined thereafter to baseline levels (Fig. 5A), while Fgflo mRNA expression was highest at day 2 and declined thereafter. This expression pattern is similar to the lung mesenchyme in vivo (Fig. 4C). In contrast, $\mathrm{Fgf7}$ mRNA expression increased over time in the 
A

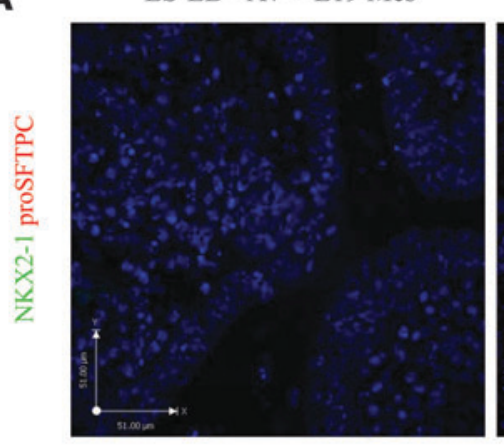

iPS-EB +AV + E19 Mes

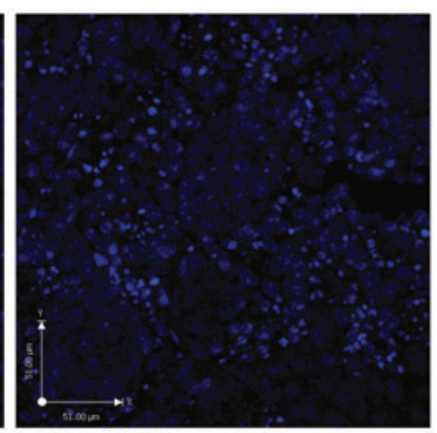

B

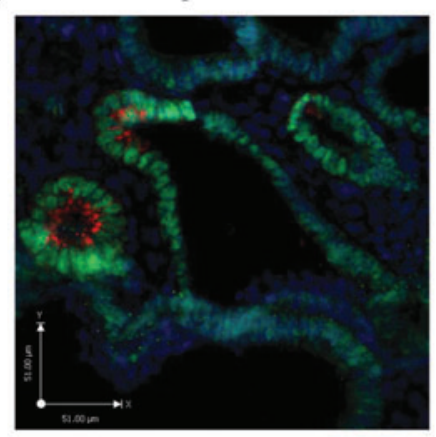

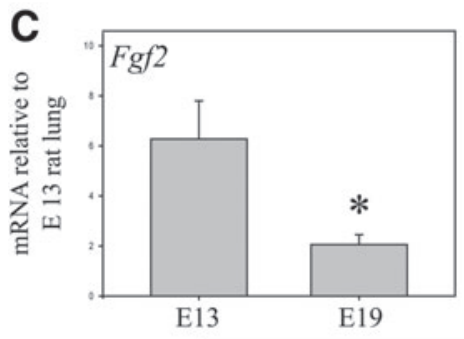

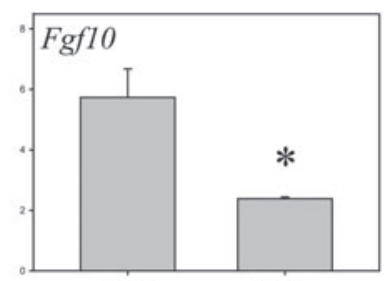

E13

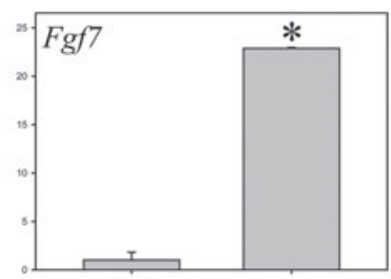

E13

E19

Mesenchyme

E

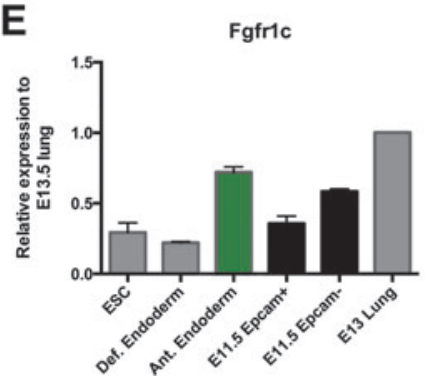

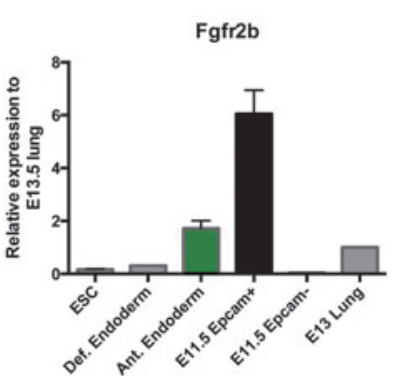
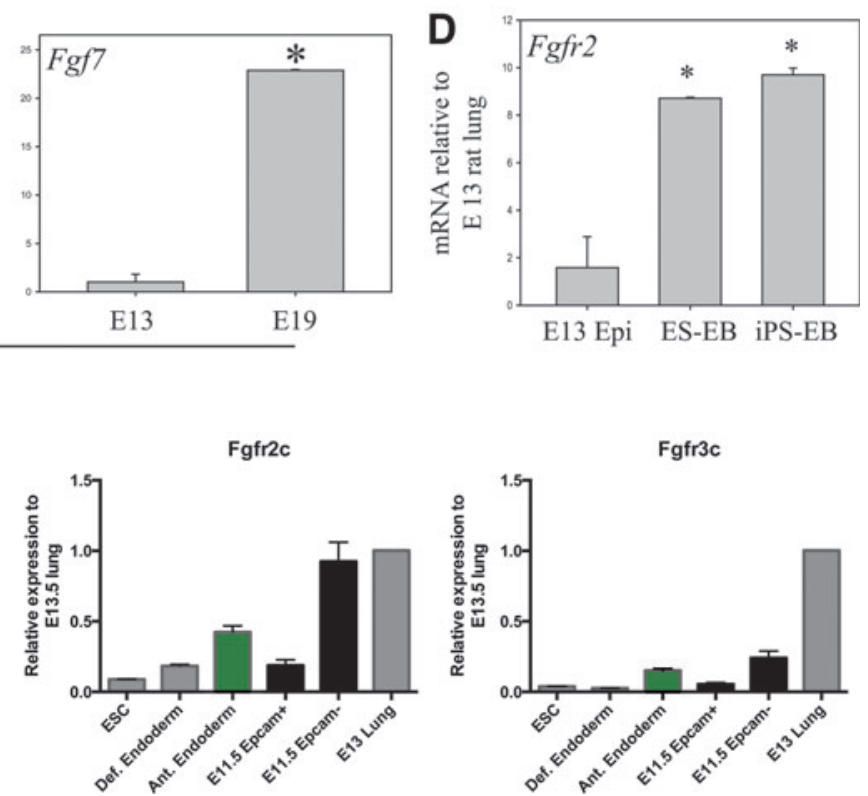

E13 Epi ES-EB iPS-EB

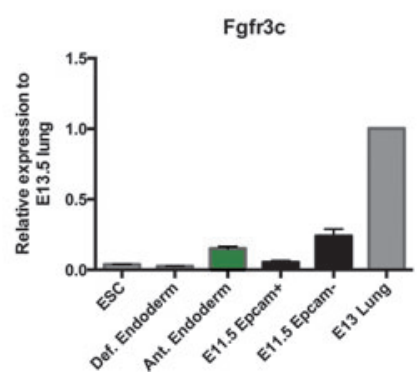

FIG. 4. Fgf expression in early and late-gestation lung mesenchyme. (A) Lung mesenchyme of late gestation (E19) is unable to induce an early lung cell (NKX2-1 ${ }^{+} /$proSFTPC $^{+}$) phenotype in endoderm-induced ESCs or iPSCs. Positive (B) immunoreactivity for NKX2-1 and proSFTPC was noted when E13 lung epithelium was combined with E19 mesenchyme. Epi, epithelium. (C) Developmental mRNA expression pattern of $F g f 2,7$, and 10 as assessed by qPCR in early (E13) and late (E19) rat lung mesenchyme. (D) Both ESCs and iPSCs express Fgfr2 mRNA at significantly higher levels than E13 epithelium. Values are means \pm SEM $(n=3, * P<0.05)$. (E) Differential expression pattern of various $F g f r$ transcripts (Fgfrlc, Fgfr2b, Fgfr2c, and Fgfr3c) in early (E11.5) embryonic lung tissues and prelung endoderm cells (definitive endoderm and anterior foregut endoderm). $\mathrm{EPCAM}^{+}$(epithelial) and $\mathrm{EPCAM}^{-}$(mesenchymal) cell populations were isolated from E11.5 mouse lung buds. Values are means \pm SEM $(n=3)$. Green bar, anterior endoderm; black bars, sorted EPCAM + or EPCAM - E11.5 lung cell populations. FGF, fibroblast growth factor; qPCR, quantitative PCR. Color images available online at www.liebertpub.com/scd

EB-mesenchyme aggregates (Fig. 5A), a pattern that also mimics what occurs in the lung mesenchyme in vivo (Fig. 4C). Nkx2-1 mRNA expression appears to follow the expression pattern of $F g f 7$, as it steadily increased between days 2 and 6 in both ESC and iPSC aggregate cultures (Fig. 5B).

\section{FGF receptor expression in endoderm-induced ESCs}

Five FGF receptors have been identified to date but only the FGFR2 receptor has been shown to be crucial for lung development [45]. The FGFR2 receptor has two primary isoforms. FGFR2b is expressed primarily in the epithelium and binds mesenchymal-derived FGF7 and FGF10, which are key regulators of lung branching, proliferation, and differentiation throughout development $[32,43,46]$. FGFR2c is expressed in the mesenchyme and epithelial cells in early lung development [47] and binds FGF2, which is involved in early specification of endoderm and lung cell lineages [1], and FGF9, which is important for expansion of the mesenchymal and epithelial cell compartments [48]. We first confirmed the mRNA expression profile of both Fgfr $2 b$ and $\mathrm{Fg} f \mathrm{r} 2 \mathrm{c}$ in sorted $\mathrm{EPCAM}^{+}$(epithelial) and $\mathrm{EPCAM}^{-}$ (mesenchymal) cells of E11.5 mouse lungs (Fig. 4E); the epithelial compartment showed high expression of $F g f r 2 b$ and relatively low expression of $F g f r 2 c$. Transcripts for Fgfr $2 c$ were detected mostly in the mesenchyme. Both definitive endoderm-induced ESCs and iPSCs expressed total Fgfr2 mRNA (Fig. 4D). Additional real-time PCR using isoform-specific primers revealed that anterior-endodermal cells derived from ESCs expressed significant levels of Fgfr2b mRNA. Expression levels of Fgfr2c and Fgfrlc were much lower while that of $F g f 3 c$ were negligible (Fig. 

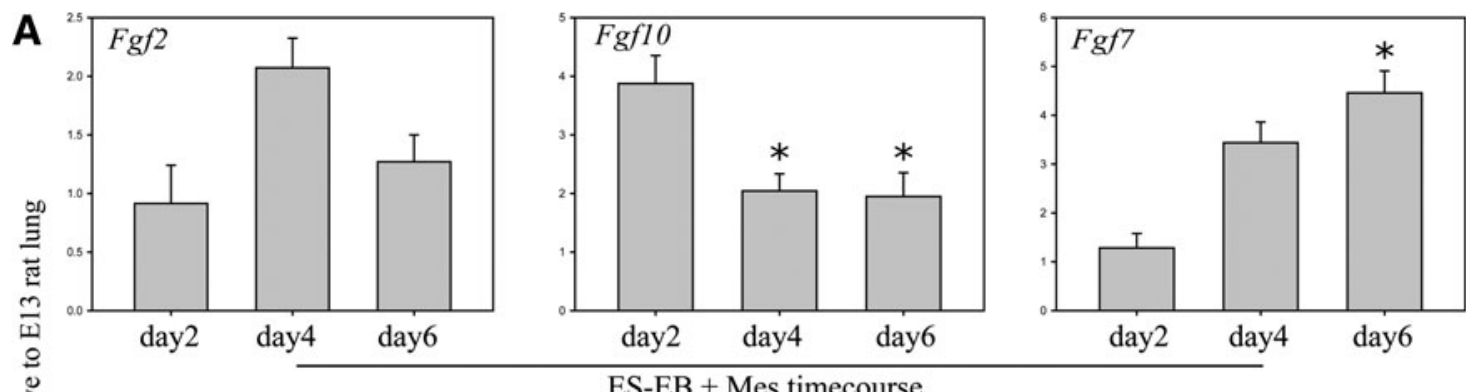

ES-EB + Mes timecourse

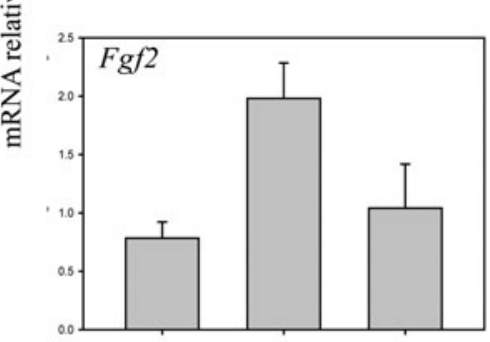

day2 day4 day6

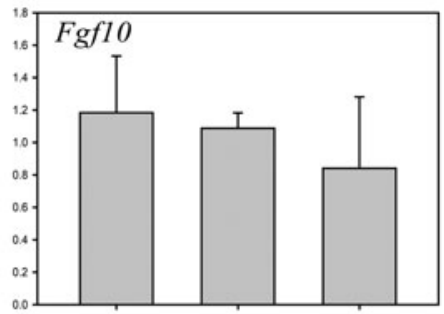

day2 day4 day6

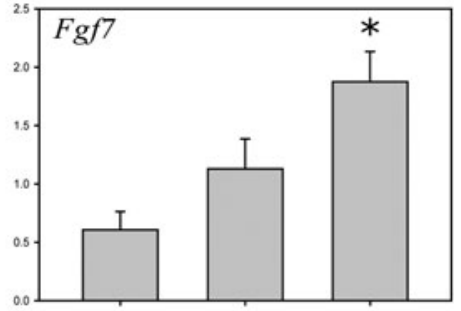

day2 day4 day6

iPS-EB + Mes timecourse

B

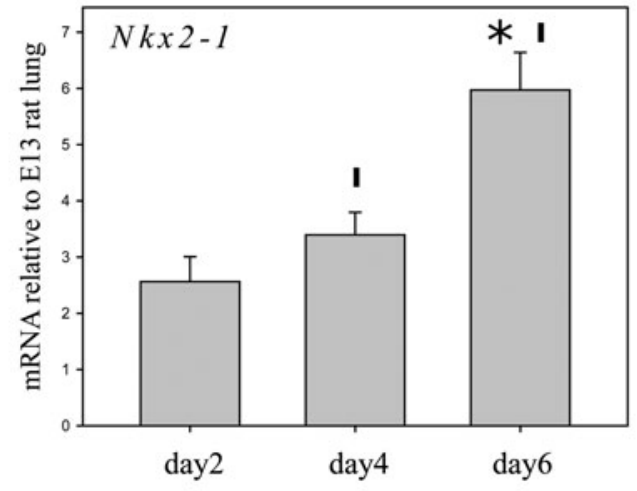

ES-EB + Mes timecourse

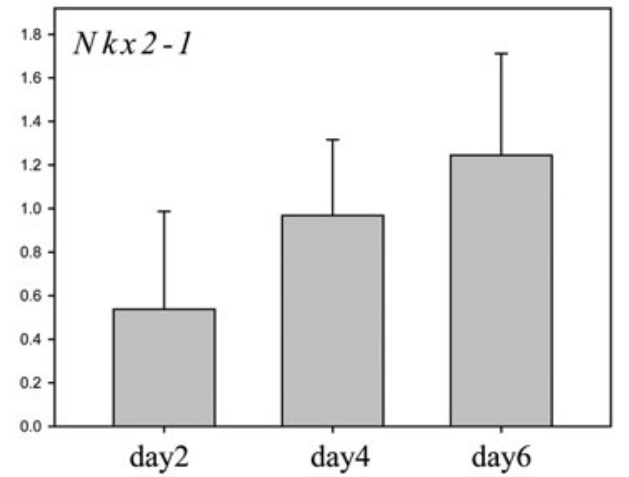

iPS-EB + Mes timecourse

FIG. 5. Temporal expression of $F g f s$ and $N k x 2-1$ in mesenchyme-EB aggregates. Expression was measured by qPCR of EB-mesenchyme aggregates at days 2, 4, and 6 in culture. (A) Fgf2 mRNA expression peaked at day 4 and declined thereafter in both ES and iPS cultures. Fgflo mRNA expression decreased with duration of culture while Fgf7 expression increased. (B) Expression of $N k x 2-1$ mRNA increased with duration of time in a pattern similar to $F g f 7$. Values are mean $\pm \operatorname{SEM}\left(n=3\right.$ separate experiments in duplicate); ${ }^{*} P<0.05$. Asterisks denote significance against EB-Mes aggregates at day 4, while cross denotes significance against E13 control lung.

4E). These data suggest that anterior endoderm-induced ESCs and iPSCs are capable of responding directly to various FGF signaling, including FGF2, FGF7, FGF9, and FGF10.

\section{FGFR2b signaling is not crucial for differentiation into early lung progenitor cells}

Having confirmed Fgfrlc, Fgfr $2 b$, and Fgfr $2 c$ expression in anterior endoderm-induced ESCs and iPSCs, and that of $F g f 2,7$, and 10 in early lung mesenchyme, we next examined the contribution of these FGFs for initial commitment to NKX2-1/proSFTPC-positive cells using truncated soluble receptors. We treated the aggregates with a high concentration of truncated soluble FGFR2b (sFGFR2b) to determine whether disruption of signaling through this receptor isoform will affect differentiation. This resulted in limited

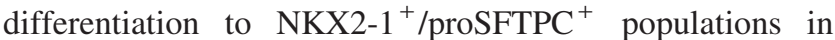
small regions of the ESC- and iPSC-derived aggregates (Fig. 6A) that lacked tubular organization when compared with nontreated controls. Soluble NGFR, a structural analogue to the soluble FGF receptors, was used as a control as it is not known to play a role in distal lung epithelial differentiation. Immunoblotting provided further evidence as a weaker positive signal for both NKX2-1 and proSFTPC was detected in the aggregates treated with sFGFR2b (Fig. 6B). Treatment with truncated soluble FGFR2c (sFGFR2c) completely eliminated the $\mathrm{NKX} 2-1^{+} /$proSFTPC $^{+}$populations as demonstrated by immunostaining and immunoblotting in both ESC- and iPSC-derived aggregates (Fig. 6A, B). The addition of both truncated soluble receptors together showed a similar result, with no expression of any lung cell markers and an overall lack of organization within the cultures. To test the specificity of the blocking receptor 
A
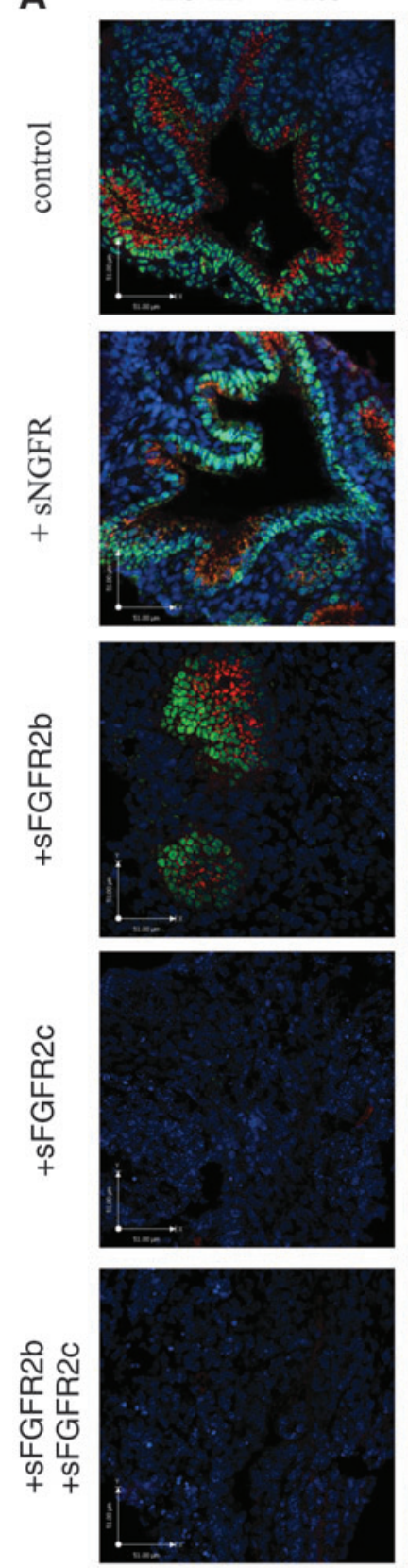

NKX2-1 proSFTPC
iPS EB + Mes
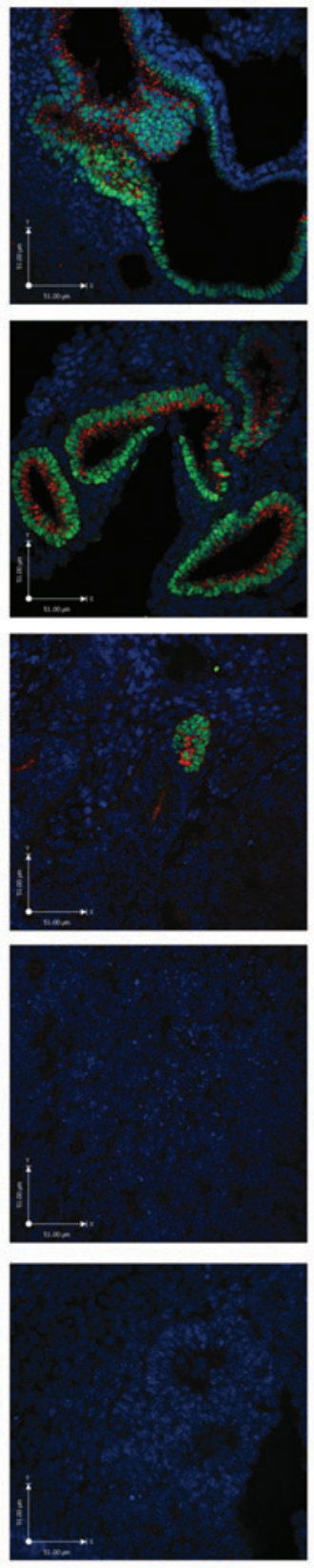
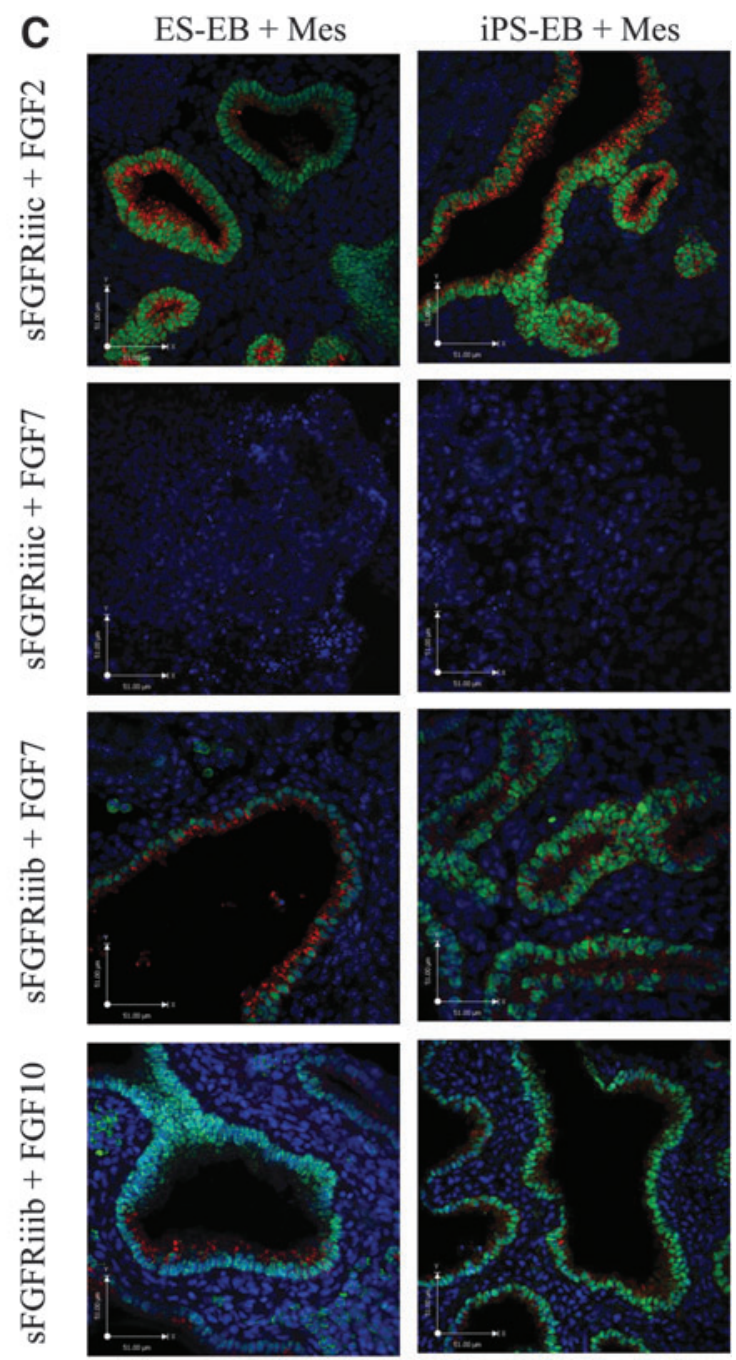

NKX2-1 proSFTPC

B

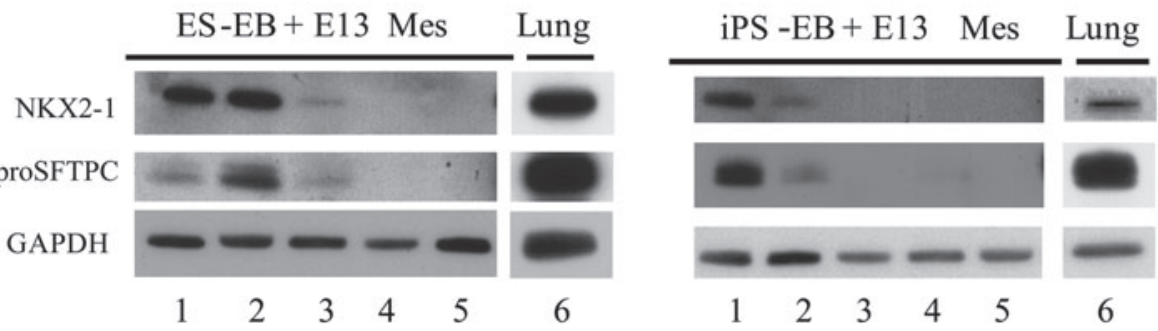

FIG. 6. Blockage of signaling through FGFR2c, but not FGFR2b, inhibits ESC and iPSC differentiation to an NKX2-1/ proSFTPC-positive lineage. (A) NKX2-1 (green)/proSFTPC (red) immunostaining of ESCs and iPSCs containing aggregates treated with or without soluble FGFR2 receptors (sNGFR, soluble receptor control). Positive NKX2-1/proSFTPC immunoreactivity was completely inhibited by sFGFR2c, while some positive clusters were still noted in the sFGFR2b-treated aggregates. (B) Immunoblotting of aggregate lysates confirmed immunostaining data: lane 1, untreated control; lane 2, sNGFR control; lane 3, sFGFR2b; lane 4, sFGFR2c; lane 5, sFGFR2b/FGFR2c; lane 6, adult lung control. (C) Immunostaining shows that outcompeting the soluble FGFR2c with FGF2, but not FGF7, restored expression of NKX2-1 (green) and proSFTPC (red) as well as structural organization within the aggregates. Addition of FGF7 and FGF10 also restores the expression of NKX2-1/ proSFTPC in aggregates treated with sFGFR2b. Color images available online at www.liebertpub.com/scd 


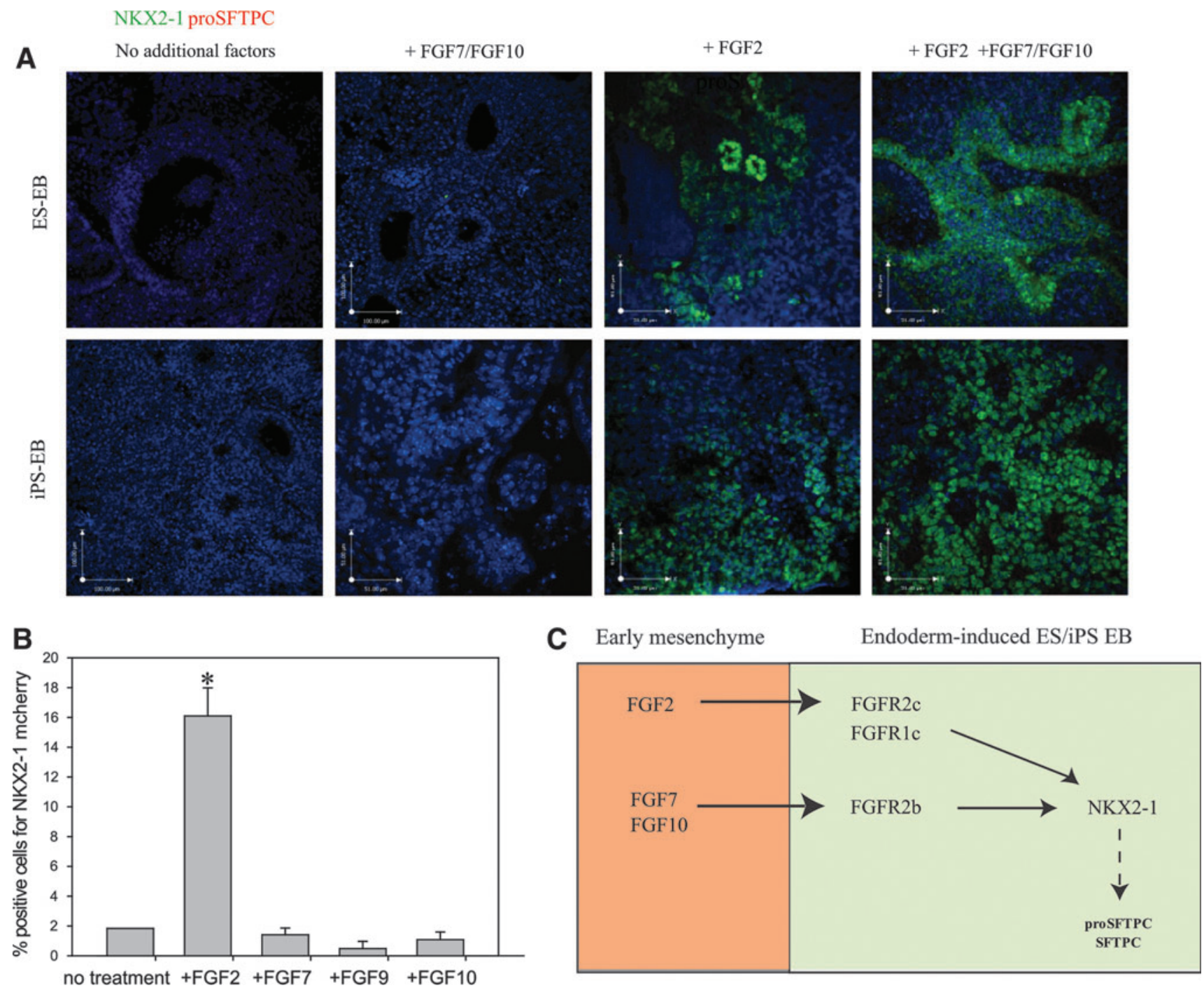

FIG. 7. Addition of FGF2 to endoderm $\left(\mathrm{EPCAM}^{+} / \mathrm{CXCR}^{+}{ }^{+}\right)$-sorted cells induces an NKX2-1 ${ }^{+}$phenotype. (A) Sorted ES and iPS endoderm clusters cultured at the air-liquid interface without mesenchyme do not express NKX2-1 or proSFTPC. The addition of $100 \mathrm{ng} / \mathrm{mL}$ of either FGF7 or -10 did not result in any positive expression of NKX2-1. In the presence of $250 \mathrm{ng} / \mathrm{mL}$ of FGF2 a subset of sorted cells expressed NKX2-1, while proSFTPC was never detected. The addition of 100-250 ng/mL of FGF7 and FGF10 to the FGF2 conditions increased the proportion of NKX2-1 ${ }^{+}$cells, but did not further enhance differentiation and proSFTPC was not detected. Green, NKX2-1; red, proSFTPC; blue, DAPI. (B) Flow cytometry analysis of NKX2-1 ${ }^{\text {mcherry }}$-sorted cells treated with the various FGFs confirmed that only FGF2 was able to induce NKX2-1 expression in the cell aggregates above control levels. $* P<0.05$, asterisks denotes significance against No Treatment group. (C) Schematic of signaling interactions in commitment of ESCs and iPSCs to a lung cell phenotype in mesenchymal-EB aggregates. FGF2 signals through the FGFR1c and/or FGFR2c receptors on the endoderm-induced cells to induce NKX2-1 expression in ES and iPS cells, while FGF7 and FGF10 that signal through the FGFR2b receptor are unable to induce expression of NKX2-1. Color images available online at www.liebertpub.com/scd

isoforms, we out-competed the soluble FGFR receptors with exogenous FGFs. Addition of FGF2, which signals through FGFR1c and FGFR2c, restored differentiation to an NKX2$1^{+} /$proSFTPC $^{+}$phenotype in aggregate cultures treated with sFGFR2c (Fig. 6C). In contrast, addition of FGF7, which has negligible binding affinity for FGFR1c and FGFR2c [49], did not reverse the negative effects of sFGFR2c on NKX2-1/proSFTPC expression (Fig. 6C). Both FGF7 and -10 bind with high affinity to FGFR2b [49] and, indeed, their addition to the aggregate cultures treated with sFGFR2b enhanced the expression of NKX2-1/proSFTPC and restored the organization into tubular structures. Together, this data suggests that the mesenchymal induction of
ESC- and iPSC-derived definitive endoderm toward NKX2$1^{+} /$proSFTPC $^{+}$lung progenitor cells likely involves FGFR1c and/or FGFR2c, but not FGFR2b, signaling.

\section{FGF2 is unable to mimic the full effects of early lung mesenchyme}

Concurrent with our soluble FGFR2c findings, other studies [12,31] have demonstrated that FGF2 (a FGFR1c/FGFR2c ligand) is responsible for commitment of endoderm-induced cells to an NKX2-1 ${ }^{+}$lineage. To examine the induction capacity of FGF2 alone in our culture system, we sorted activin A-treated iPSCs and ESCs for $\mathrm{EPCAM}^{+} / \mathrm{CXCR}^{+}$ 
cells, which were then plated at air-liquid interface on floating membranes, without mesenchyme. Sorted cell clusters cultured without any factors did not express NKX21 or proSFTPC, nor did the addition of FGF7 or FGF10 (alone or together) induce lung marker expression. Addition of FGF2, however, resulted in large clusters of NKX2-1 ${ }^{+}$ cells, although lacking organization. Further addition of FGF7 and FGF10 after 3 days of FGF2 exposure resulted in expansion of the NKX2-1 ${ }^{+}$cell population. ProSFTPC expression was not present at any concentration of FGF2 or in combination with FGF7 and FGF10 (Fig. 7A). Only cells treated with FGF2 expressed levels of NKX2-1 $(\sim 16 \%)$ above untreated controls quantified with flow cytometry using Nkx2-1 ${ }^{\text {mCherry }}$ ES cells. FGF9, which exhibits a high affinity for FGFR3c but also binds (although with lower affinity) to FGFR2c and FGFR1c [49], was unable to induce any significant levels of NKX2-1 (Fig. 7B). Overall, this provides evidence that complex FGF signaling is required to commit endoderm-induced pluripotent cells to an NKX2-1 ${ }^{+}$ lineage, but is not sufficient to efficiently induce a distal lung phenotype characterized by the coexpression of NKX21 and proSFTPC.

\section{Discussion}

In this study, using lung mesenchyme, we were able to rapidly induce ESCs and iPSCs to a distal lung epithelial cell phenotype with high efficiency. We built on the initial ESC work by Bishop and colleagues [9] by utilizing the known inductive capabilities of the embryonic mesenchyme [2-4]. We first treated ESCs and iPSCs with activin A, which were then sorted to obtain a highly enriched endoderm population. The sorted cells were subsequently combined and cultured as 3D aggregates with embryonic (E13) lung mesenchyme. Within 10-11 days we were able to generate a population of polarized lung epithelial cells from ESCs and iPSCs that were 80\% positive for NKX2-1 and largely expressing proSFTPC, making this to our knowledge, one of the most rapid and efficient inductions of pluripotent cells into distal lung epithelial progenitor cells to date $[10,12,13]$. We found coexpression of hCD4 and EPCAM with a separate ES cell line at similar levels. The starting ratio of endoderm-sorted stem cells to mesenchyme remained roughly $30 \%$ following 6 days in aggregate culture, despite a 5-fold increase in total cell number. This suggests that the endoderm-induced stem cells and mesenchymal cells proliferate at similar rates and the majority of the endoderm-induced stem cells retain EPCAM expression following sorting, aggregation, and culture with mesenchyme.

Surfactant protein B expression was detected in both ESCs and iPSCs containing aggregates at greater levels than E13 lung. Although Rippon et al. have examined Sftpb expression, the data were normalized to ESCs and, therefore, it is unknown how this expression relates to the early lung [13]. Huang et al. recently reported directed differentiation of human ESCs in distal lung cells with $\sim 50 \%$ of cells expressing SFTPB [11]. However, only 5\% of the cells expressed SFTPC, the typical marker for type II cells [42], indicating that no fully differentiated alveolar epithelial phenotype was achieved. No expression of SCGB1A1 (Club/Clara cell marker) or SOX2 (proximal lung progenitor marker when coexpressed with NKX2-1) was found in any of our culture conditions, in line with previous studies that used recombinants of early distal lung mesenchyme with embryonic lung epithelium [32]. The observed colocalization of SOX9 expression (distal lung progenitor marker when coexpressed with NKX2-1) with proSFTPC and NKX2-1 underscores the distal inductive capacity of the mesenchyme [3,32]. Expression patterns of $F g f s$ in the EBmesenchyme aggregate cultures mimicked those of the developing lung $[43,44]$. The temporal expression pattern of mesenchymal-derived Fgfs when cultured with ESC- and iPSC-derived endoderm suggests that the mesenchyme is not simply acting as a static provider of signals, but that communication in the form of crosstalk occurs between the two tissue layers in a three-dimensional setting. Interestingly, while developmental studies that used late embryonic (E19) mesenchyme found that these cells can support early embryonic epithelial cell growth and differentiation [32], late mesenchyme was unable to specify ESCs or iPSCs to an NKX2 $-1^{+}$lineage. This suggests that early lung epithelium is already more lineage committed than the endoderminduced ESCs and iPSCs and is better primed to respond to factors present in the late lung mesenchyme.

We provide evidence that signaling through FGFR1c and/or FGFR2c is critical for commitment of ESCs and iPSCs to an NKX2 $-1^{+}$phenotype, and that FGF2 can initiate this event. Previous studies that used $F g f r 2 b$-null mice, with intact FGFR2c function, show that the mice are viable until birth; however, they suffer from severe defects of the limbs, lungs, and the posterior pituitary gland [50]. In these mice, the development of the lung is initiated, as a primitive lung bud can be found, but fails to continue to develop and differentiate appropriately, eventually undergoing extensive apoptosis. This finding is consistent with our results, as signaling via FGFR2c (and/or FGFR1c) seems to be critical for the initial induction of NKX2-1 expression, whereas FGFR2b appears to play a role in organization, and further proliferation and differentiation. FGFR1c, the functionally active isoform of FGFR1 [51], is expressed throughout the epithelium and mesenchyme of the early chick lung [52], in agreement with our Fgfrlc expression findings in E11.5 $\mathrm{EPCAM}^{+}$(epithelium) and $\mathrm{EPCAM}^{-}$ (mesenchymal) cells. Fgfrl-null mice have demonstrated essential functions in embryonic development prior to early lung formation $[51,53]$. Although a role for FGFR1c in the developing lung mesenchymal has been demonstrated [48,54], the importance of epithelial FGFR1c is unknown.

Our observation that FGF2, but not FGF7, is able to "rescue" the NKX2.1/proSFTPC induction in 3D aggregates treated with sFGFR2c shows the specificity of the receptor isotypes and suggests that FGF2 is a key molecule responsible for initial commitment to an early lung epithelial cell phenotype. In vitro studies have shown that FGF2 signaling from the primitive foregut mesenchyme is able to induce endoderm organ formation at varying concentrations, with higher concentrations specifying lung and lower concentrations specifying pancreas and liver [1]. Further support for our findings comes from early tracheal epithelial cultures in the absence of mesenchyme that assessed the potential of various FGFs to reprogram the proximal epithelium to a distal phenotype expressing SFTPC [55]. FGF2 was able to induce an SFTPC phenotype. Studies with ESCs have shown conflicting results on the effects of FGF2 on distal lung cell differentiation with some showing that it decreases alveolar type II cell markers [56] while others have reported that it is 
necessary for commitment to a proSFTPC ${ }^{+}$population [10]. Fgf2-null mice have a relatively mild phenotype [57,58], which has led to speculation that there is compensation for FGF2 functions by FGF1; however, Fgfl;Fgf2 double knockouts produce a similar mild phenotype [59]. This suggests that either other factors (including other FGFs) are taking over the roles of FGF1 and FGF2, or that they play limited role in lung development and homeostasis. When we examined the inductive capacity of FGF2 alone on endoderm-induced ESCs and iPSCs, limited patches of NKX2$1^{+}$cells lacking proSFTPC expression were observed. The overall percentage of cells expressing NKX2-1 in these monolayer cultures was much lower than those of cells cultured as 3D aggregates with early lung mesenchyme (16\% vs. $80 \%$ ). This confirms that while FGF2 is important for the initial specification, it is not sufficient. Additional FGFs are required (see schematic depicted in Fig. 7C). Moreover, numerous other factors, including matrix proteins, aid in the polarization, differentiation, and maturation to mature lung epithelia [60]. Interestingly, FGF9 was unable to induce an NKX2-1 lineage in endoderm-induced ESCs. FGF9 signaling via mesenchymal FGFR1c and FGFR2c has been shown to be important for lung mesenchymal proliferation $[48,61]$. However, treatment of the 3D aggregates with sFGFR2c did not result in significantly smaller aggregates with less mesenchyme, suggesting that FGF9 signaling in the aggregates did not occur or was not completely abolished by sFGFR2c. Fgf9 mRNA expression increased in the 3D aggregates with duration in culture (not shown); thus, the amount of sFGFR2c used may not have inhibited FGF9 signaling. Other studies have suggested that FGF9 acts on epithelial proliferation via FGFR2b [62]; however, FGF9 addition to aggregates treated with sFGFR2b did not the overcome the antiproliferative action of sFGFR2b (not shown). While the precise binding site of the ligands to FGFRs has yet to be characterized, there is evidence that cofactors, including heparin, and the formation of heterodimers between FGFs and their receptors can alter binding specificity and activation of downstream pathways in vivo [44,63], which may explain in part why FGF2 and FGF9 did not produce the same result in the endoderm-induced pluripotent cells.

The importance of crosstalk between tissue layers for proper lung morphogenesis and differentiation is well accepted. During early lung development, the mesenchyme has been shown to be the instructive tissue layer [2$4,22,32]$, and in this study we have attempted to build on this knowledge and translate it to the differentiation of ES and iPS cells. While mesenchyme has been used previously to differentiate ES cells [9], it had not been fully characterized with respect to differentiation potential or efficiency, nor has it been done using an optimized starting material. The underlying hypothesis is that early lung mesenchyme has the ability to differentiate ES and iPS cells to an early lung cell phenotype, and that this lineage commitment is due to FGF signaling. As expected, early lung mesenchyme supported the differentiation of pluripotent cells into early lung progenitor cells and this effect was mediated through the FGFR1c and/or FGFR2c receptors. Our success in differentiating mouse ESCs and iPSCs using both rat and mouse lung mesenchyme illustrates the conserved nature and importance of the FGF pathway in early cell fate decisions $[64,65]$. While others have attempted to differentiate
ESCs [10-12] or iPSCs [20] in a monolayer in culture, such an approach does not take into account the complexities of the cell-cell and cell-matrix interactions as well as the 3D spatial environment. Moreover, since all of the nuances of lung development have yet to be characterized, including cell intermediates and selection of the compliment of factors, their concentration and optimal timing of administration is a matter of trial and error. Aggregate cultures with mesenchyme overcome many of these issues as the appropriate factors and dosages are provided, including the matrix proteins, reciprocal signaling, and a 3D environment, which contribute to an optimal induction milieu. The rapid and efficient differentiation as well as the morphogenesis seen in this culture system demonstrates that generating lung epithelial cells from a pluripotent starting material requires a system that can temporally adapt to the needs of the differentiating cells. Our current findings highlight the need for more complex protocols that focus on matrix interactions, growth factors, and reciprocal signaling of the differentiating cells in place of archaic monolayer culture systems.

\section{Acknowledgments}

The authors wish to thank Dr. Ellis for supplying the iPS cells, Dr. Keller for the Foxa2/hCD4;Bry/GFP ESC, and Dr. Rossant for the Nkx2-1 ${ }^{\text {mcherry }}$ ESCs. FACS was performed in The SickKids-UHN Flow Cytometry Facility. This work was supported by operating grants (MOP-77751 and RMF92088) from the Canadian Institutes for Health Research and an infrastructure grant (CSCCD) from the Canadian Foundation of Innovation. E.F. was the recipient of a CIHR Banting and Best doctoral award. M.B. received a CIHR/Canadian Lung Association/GlaxoSmithKline partnership fellowship and a fellowship from the Fonds de la Recherche en Santé $d u$ Québec. M.P. is the holder of a Canadian Research Chair in Fetal, Neonatal and Maternal Health.

\section{Author Disclosure Statement}

No competing financial interests exist.

\section{References}

1. Serls AE. (2004). Different thresholds of fibroblast growth factors pattern the ventral foregut into liver and lung. Development 132:35-47.

2. Masters JR. (1976). Epithelial-mesenchymal interaction during lung development: the effect of mesenchymal mass. Dev Biol 51:98-108.

3. Shannon JM, LD Nielsen, SA Gebb and SH Randell. (1998). Mesenchyme specifies epithelial differentiation in reciprocal recombinants of embryonic lung and trachea. Dev Dyn 212:482-494.

4. Spooner BS and NK Wessells. (1970). Mammalian lung development: interactions in primordium formation and bronchial morphogenesis. J Exp Zool 175:445-454.

5. D'Amour KA, AG Bang, S Eliazer, OG Kelly, AD Agulnick, NG Smart, MA Moorman, E Kroon, MK Carpenter and EE Baetge. (2006). Production of pancreatic hormoneexpressing endocrine cells from human embryonic stem cells. Nat Biotechnol 24:1392-1401.

6. Gouon-Evans V, L Boussemart, P Gadue, D Nierhoff, CI Koehler, A Kubo, DA Shafritz and G Keller. (2006). BMP- 
4 is required for hepatic specification of mouse embryonic stem cell-derived definitive endoderm. Nat Biotechnol 24:1402-1411.

7. Kroon E, LA Martinson, K Kadoya, AG Bang, OG Kelly, S Eliazer, H Young, M Richardson, NG Smart, et al. (2008). Pancreatic endoderm derived from human embryonic stem cells generates glucose-responsive insulin-secreting cells in vivo. Nat Biotechnol 26:443-452.

8. Ali NN, AJ Edgar, A Samadikuchaksaraei, CM Timson, HM Romanska, JM Polak and AE Bishop. (2002). Derivation of type II alveolar epithelial cells from murine embryonic stem cells. Tissue Eng 8:541-550.

9. Van Vranken BE, HM Romanska, JM Polak, HJ Rippon, JM Shannon, AE Bishop. (2005). Coculture of embryonic stem cells with pulmonary mesenchyme: a microenvironment that promotes differentiation of pulmonary epithelium. Tissue Eng 11:1177-1187.

10. Roszell B, MJ Mondrinos, A Seaton, DM Simons, SH Koutzaki, G Fong, PI Lelkes, CM Finck. (2009). Efficient derivation of alveolar type II cells from embryonic stem cells for in vivo application. Tissue Eng Part A 15:3351-3365.

11. Huang SXL, MN Islam, J O'Neill, Z Hu, Y-G Yang, Y-W Chen, M Mumau, MD Green, G Vunjak-Novakovic, J Bhattacharya and H-W Snoeck. (2013). Efficient generation of lung and airway epithelial cells from human pluripotent stem cells. Nat Biotechnol 32:84-91.

12. Longmire TA, L Ikonomou, F Hawkins, C Christodoulou, Y Cao, JC Jean, LW Kwok, H Mou, J Rajagopal, et al. (2012). Efficient derivation of purified lung and thyroid progenitors from embryonic stem cells. Cell Stem Cell 10:398-411.

13. Rippon HJ, JM Polak, M Qin and AE Bishop. (2006). Derivation of distal lung epithelial progenitors from murine embryonic stem cells using a novel three-step differentiation protocol. Stem Cells 24:1389-1398.

14. Wang D, DL Haviland, AR Burns, E Zsigmond and RA Wetsel. (2007). A pure population of lung alveolar epithelial type II cells derived from human embryonic stem cells. Proc Natl Acad Sci U S A 104:4449-4454.

15. Winkler ME, C Mauritz, S Groos, A Kispert, S Menke, A Hoffmann, I Gruh, K Schwanke, A Haverich and U Martin. (2008). Serum-free differentiation of murine embryonic stem cells into alveolar type II epithelial cells. Cloning Stem Cells 10:49-64.

16. Coraux C, B Nawrocki-Raby, J Hinnrasky, C Kileztky, D Gaillard, C Dani and E Puchelle. (2005). Embryonic stem cells generate airway epithelial tissue. Am J Respir Cell Mol Biol 32:87-92.

17. Mou H, R Zhao, R Sherwood, T Ahfeldt, A Lapey, J Wain, L Sicilian, K Izvolsky, FH Lau, et al. (2012). Generation of multipotent lung and airway progenitors from mouse ESCs and patient-specific cystic fibrosis iPSCs. Cell Stem Cell 10:385-397.

18. Nishimura Y, TS Hamazaki, S Komazaki, S Kamimura, H Okochi and M Asashima. (2006). Ciliated cells differentiated from mouse embryonic stem cells. Stem Cells 24:1381-1388.

19. Ghaedi M, EA Calle, JJ Mendez, AL Gard, J Balestrini, A Booth, PF Bove, L Gui, ES White and LE Niklason. (2013). Human iPS cell-derived alveolar epithelium repopulates lung extracellular matrix. J Clin Invest 123:4950-4962.

20. Wong AP, CE Bear, S Chin, P Pasceri, TO Thompson, L-J Huan, F Ratjen, J Ellis and J Rossant. (2012). Directed differentiation of human pluripotent stem cells into mature airway epithelia expressing functional CFTRTR protein. Nat Biotechnol 30:876-882.
21. Kadzik RS and EE Morrisey. (2012). Directing lung endoderm differentiation in pluripotent stem cells. Cell Stem Cell 10:355-361.

22. Shannon JM. (1994). Induction of alveolar type II cell differentiation in fetal tracheal epithelium by grafted distal lung mesenchyme. Dev Biol 166:600-614.

23. D'Amour KA, AD Agulnick, S Eliazer, OG Kelly, E Kroon and EE Baetge. (2005). Efficient differentiation of human embryonic stem cells to definitive endoderm. Nat Biotechnol 23:1534-1541.

24. Kubo A, S Katsunori, JM Shannon, V Kouskoff, M Kennedy, S Woo, HJ Fehling and G Keller. (2004). Development of definitive endoderm from embryonic stem cells in culture. Development 131:1651-1662.

25. Zhou X, H Sasaki, L Lowe, BLM Hogan and MR Kuehn. (1993). Nodal is a novel TGF-b-like gene expressed in the mouse node during gastrulation. Nature 361:543-547.

26. Hotta A, AYL Cheung, N Farra, K Garcha, WY Chang, P Pasceri, WL Stanford and J Ellis. (2009). EOS lentiviral vector selection system for human induced pluripotent stem cells. Nat Protoc 4:1828-1844.

27. Nagy A, J Rossant, R Nagy, W Abramow-Newerly and JC Roder. (1993). Derivation of completely cell culturederived mice from early-passage embryonic stem cells. Proc Natl Acad Sci U S A 90:8424-8428.

28. Gertsenstein M, LMJ Nutter, T Reid, M Pereira, WL Stanford, J Rossant and A Nagy. (2010). Efficient generation of germ line transmitting chimeras from C57BL/6N ES cells by aggregation with outbred host embryos. PLoS One 5:e11260.

29. Hotta A, AYL Cheung, N Farra, K Vijayaragavan, CA Séguin, JS Draper, P Pasceri, IA Maksakova, DL Mager, et al. (2009). Isolation of human iPS cells using EOS lentiviral vectors to select for pluripotency. Nat Methods 6: 370-376.

30. Bilodeau M, S Shojaie, C Ackerley, M Post and J Rossant. (2014). Identification of a Proximal Progenitor Population from Murine Fetal Lungs with Clonogenic and Multilineage Differentiation Potential. Stem Cell Reports 3:1-16.

31. Green MD, A Chen, M-C Nostro, SL d'Souza, C Schaniel, IR Lemischka, V Gouon-Evans, G Keller and H-W Snoeck. (2011). Generation of anterior foregut endoderm from human embryonic and induced pluripotent stem cells. Nat Biotechnol 29:267-272.

32. Deimling $\mathrm{J}, \mathrm{K}$ Thompson, I Tseu, J Wang, R Keijzer, AK Tanswell and M Post. (2007). Mesenchymal maintenance of distal epithelial cell phenotype during late fetal lung development. Am J Physiol Lung Cell Mol Physiol 292:L725-L741.

33. Ridsdale R and M Post. (2004). Surfactant lipid synthesis and lamellar body formation in glycogen-laden type II cells. Am J Physiol Lung Cell Mol Physiol 287:L743-L751.

34. Livak KJ and TD Schmittgen. (2001). Analysis of relative gene expression data using real-time quantitative PCR and the 2(-Delta Delta C(T)) method. Methods 25:402-408.

35. McGrath KE, AD Koniski, KM Maltby, JK McGann and J Palis. (1999). Embryonic expression and function of the chemokine SDF-1 and its receptor, CXCR4. Dev Biol 213:442-456.

36. Sherwood RI, C Jitianu, O Cleaver, DA Shaywitz, JO Lamenzo, AE Chen, TR Golub and DA Melton. (2007). Prospective isolation and global gene expression analysis of definitive and visceral endoderm. Dev Biol 304:541-555.

37. Perl A-KT, SE Wert, A Nagy, CG Lobe and JA Whitsett. (2002). Early restriction of peripheral and proximal cell lineages during formation of the lung. Proc Natl Acad Sci U S A 99:10482-10487. 
38. Que J, X Luo, RJ Schwartz and BLM Hogan. (2009). Multiple roles for Sox2 in the developing and adult mouse trachea. Development 136:1899-1907.

39. Guha A, M Vasconcelos, Y Cai, M Yoneda, A Hinds, J Qian, G Li, L Dickel, JE Johnson, et al. (2012). Neuroepithelial body microenvironment is a niche for a distinct subset of Clara-like precursors in the developing airways. Proc Natl Acad Sci U S A 109:12592-12597.

40. Musah S, J Chen and GW Hoyle. (2012). Repair of tracheal epithelium by basal cells after chlorine-induced injury. Respir Res 13:107.

41. Tichelaar JW, SE Wert, RH Costa, S Kimura and JA Whitsett. (1999). HNF-3/forkhead homologue-4 (HFH-4) is expressed in ciliated epithelial cells in the developing mouse lung. J Histochem Cytochem 47:823-832.

42. Weaver TE, CL Na and MT Stahlman. (2002). Biogenesis of lamellar bodies, lysosome-related organelles involved in storage and secretion of pulmonary surfactant. Semin Cell Dev Biol 13:263-270.

43. Bellusci S, J Grindley, H Emoto, N Itoh and BL Hogan. (1997). Fibroblast growth factor 10 (FGF10) and branching morphogenesis in the embryonic mouse lung. Development 124:4867-4878.

44. Shiratori M, E Oshika, LP Ung, G Singh, H Shinozuka, D Warburton, G Michalopoulos and SL Katyal. (1996). Keratinocyte growth factor and embryonic rat lung morphogenesis. Am J Respir Cell Mol Biol 15:328-338.

45. Arman E, R Haffner-Krausz, M Gorivodsky and P Lonai. (1999). Fgfr2 is required for limb outgrowth and lungbranching morphogenesis. Proc Natl Acad Sci U S A 96:11895-11899.

46. Post M, P Souza, J Liu, I Tseu, J Wang, M Kuliszewski and AK Tanswell. (1996). Keratinocyte growth factor and its receptor are involved in regulating early lung branching. Development 122:3107-3115.

47. Han RN, J Liu, AK Tanswell and M Post. (1992). Expression of basic fibroblast growth factor and receptor: immunolocalization studies in developing rat fetal lung. Pediatr Res 31:435-440.

48. White AC, J Xu, Y Yin, C Smith, G Schmid and DM Ornitz. (2006). FGF9 and SHH signaling coordinate lung growth and development through regulation of distinct mesenchymal domains. Development 133:1507-1517.

49. Zhang X, OA Ibrahimi, SK Olsen, H Umemori, M Mohammadi and DM Ornitz. (2006). Receptor specificity of the fibroblast growth factor family. The complete mammalian FGF family. J Biol Chem 281:15694-15700.

50. De Moerlooze L, B Spencer-Dene, JM Revest, M Hajihosseini, I Rosewell and C Dickson. (2000). An important role for the IIIb isoform of fibroblast growth factor receptor 2 (FGFR2) in mesenchymal-epithelial signalling during mouse organogenesis. Development 127:483-492.

51. Partanen J, L Schwartz and J Rossant. (1998). Opposite phenotypes of hypomorphic and Y766 phosphorylation site mutationsreveal a function for Fgfr1 in anteroposterior patterning of mouse embryos. Genes Dev 12:2332-2344.

52. Moura RS, JP Coutinho-Borges, AP Pacheco and J CorreiaPinto. (2011). FGF signaling pathway in the developing chick lung: expression and inhibition studies. PLoS One 6:e17660.

53. Deng CX, A Wynshaw-Boris, MM Shen, C Daugherty, DM Ornitz and P Leder. (1994). Murine FGFR-1 is required for early postimplantation growth and axial organization. Genes Dev 8:3045-3057.
54. Yin $\mathrm{Y}$, AC White, S-H Huh, MJ Hilton, H Kanazawa, F Long and DM Ornitz. (2008). An FGF-WNT gene regulatory network controls lung mesenchyme development. Dev Biol 319:426-436.

55. Hyatt BA, X Shangguan and JM Shannon. (2002). BMP4 modulates fibroblast growth factor-mediated induction of proximal and distal lung differentiation in mouse embryonic tracheal epithelium in mesenchyme-free culture. Dev Dyn 225:153-165.

56. Lin Y-M, A Zhang, A Bismarck and AE Bishop. (2010). Effects of fibroblast growth factors on the differentiation of the pulmonary progenitors from murine embryonic stem cells. Exp Lung Res 36:307-320.

57. Dono R, G Texido, R Dussel, H Ehmke and R Zeller. (1998). Impaired cerebral cortex development and blood pressure regulation in FGF-2-deficient mice. EMBO J 17:4213-4225.

58. Ortega S, M Ittmann, SH Tsang, M Ehrlich and C Basilico. (1998). Neuronal defects and delayed wound healing in mice lacking fibroblast growth factor 2. Proc Natl Acad Sci U S A 95:5672-5677.

59. Miller DL, S Ortega, O Bashayan, R Basch and C Basilico. (2000). Compensation by fibroblast growth factor 1 (FGF1) does not account for the mild phenotypic defects observed in FGF2 null mice. Mol Cell Biol 20:2260-2268.

60. Isakson BE, RL Lubman, GJ Seedorf and S Boitano. (2001). Modulation of pulmonary alveolar type II cell phenotype and communication by extracellular matrix and KGF. Am J Physiol Cell Physiol 281:C1291-C1299.

61. Colvin JS, AC White, SJ Pratt and DM Ornitz. (2001). Lung hypoplasia and neonatal death in Fgf9-null mice identify this gene as an essential regulator of lung mesenchyme. Development 128:2095-2106.

62. del Moral P-M, SP De Langhe, FG Sala, JM Veltmaat, D Tefft, K Wang, D Warburton and S Bellusci. (2006). Differential role of FGF9 on epithelium and mesenchyme in mouse embryonic lung. Dev Biol 293:77-89.

63. Uematsu F, M Kan, F Wang, JH Jang, Y Luo and WL McKeehan. (2000). Ligand binding properties of binary complexes of heparin and immunoglobulin-like modules of FGF receptor 2. Biochem Biophys Res Commun 272:830-836.

64. Glazer L and BZ Shilo. (1991). The Drosophila FGF-R homolog is expressed in the embryonic tracheal system and appears to be required for directed tracheal cell extension. Genes Dev 5:697-705.

65. Sutherland D, C Samakovlis and MA Krasnow. (1996). Branchless encodes a Drosophila FGF homolog that controls tracheal cell migration and the pattern of branching. Cell 87:1091-1101.

Address correspondence to: Dr. Martin Post

Physiology and Experimental Medicine Program Peter Gilgan Centre for Research and Learning Hospital for Sick Children 686 Bay Street

Toronto, ON M5G1X8

Canada

E-mail: martin.post@sickkids.ca

Received for publication May 7, 2014

Accepted after revision July 22, 2014

Prepublished on Liebert Instant Online July 31, 2014 